\title{
Active Sums I
}

\section{Alejandro J. Díaz-BARRIGA, Francisco GonzÁlez-Acuña, Francisco MARMOLEJO, and Leopoldo ROMÁN}

\author{
Instituto de Matemáticas \\ Universidad Nacional Autónoma de México \\ Ciudad Universitaria \\ 04510 México D.F. - México \\ diazb@math.unam.mx fico@math.unam.mx \\ quico@math.unam.mx leopoldo@math.unam.mx
}

Recibido: 26 de Junio de 2003

Aceptado: 19 de Diciembre de 2003

\begin{abstract}
Given a generating family $\mathcal{F}$ of subgroups of a group $G$, closed under conjugation and with partial order compatible with inclusion, a new group $S$ can be constructed, taking into account the multiplication in the subgroups and their mutual actions given by conjugation. The group $S$ is called the active sum of $\mathcal{F}$, has $G$ as a homomorph and is such that $S / Z(S) \simeq G / Z(G)$, where $Z$ denotes the center.

The basic question we investigate in this paper is: when is the active sum $S$ of the family $\mathcal{F}$ isomorphic to the group $G$ ?

The conditions found to answer this question are often of a homological nature. We show that the following groups are active sums of cyclic subgroups: free groups, semidirect products of cyclic groups, Coxeter groups, Wirtinger approximations, groups of order $p^{3}$ with $p$ an odd prime, simple groups with trivial Schur multiplier, and special linear groups $\operatorname{SL}_{n}(q)$ with a few exceptions.

We show as well that every finite group $G$ such that $G / G^{\prime}$ is not cyclic is the active sum of proper normal subgroups.
\end{abstract}

Key words: active sums, active sums of cyclic groups, regularity and independence, atomic and molecular groups.

2000 Mathematics Subject Classification: 20D99, 20E99, 20E15, 20J05, 08A55. 


\section{Introduction}

A motivating question for the concept of active sum is: to what extent can one recover a group $G$ from a generating family $\mathcal{F}=\left\{F_{i}\right\}_{i \in I}$ of subgroups, closed under conjugation, and their natural actions?

To be more precise, consider the disjoint union $U=\coprod_{i} F_{i}$, and the (partial) multiplication on $U$ induced by the multiplication in the subgroups ( $x y$ is defined if and only if $x$ and $y$ belong to the same subgroup in the disjoint union). One defines a second (this one global) operation, conjugation, in $U$ by $x^{y}=y^{-1} x y \in F_{k}$ where $x \in F_{i}, y \in F_{j}$, and $F_{i}^{y}=F_{k}$ (we are assuming here that the subgroups are all different; see however Remark 1.4). Thus $U$ is what we will be calling an active add, that is, a partial algebra with two binary partial operations. We think of $U$ as the family of subgroups $\left\{F_{i}\right\}_{i \in I}$ and their mutual actions, and we call it the active add determined by $\mathcal{F}$. Every active add determines a group called the active sum of $U$. This group $S$ is the image of $U$ under the left adjoint to the inclusion functor from the category of groups to the category of active adds. We show that the natural epimorphism $\varphi$ from $S$ onto $G$ is such that $\varphi^{-1}(Z(G))=Z(S)$, where $Z$ denotes the center. We want to know under what conditions the active sum is isomorphic to the group $G$. The fact that $\operatorname{ker}(\varphi) \subset Z(S)$ allows us to use homology of groups, in particular the five-term exact sequence and Ganea's extention to six terms [21], [4], [12].

The concept of active sums of groups originated in papers by F. Tomàs [43], [44] and P. Ribenboim [35]. Tomàs considered what in Ribenboim's terminology are discrete active families of normal subgroups and proved, for example, that any finite group $G$ is the active sum of the family $\left\langle W_{p}\right\rangle$, where $p$ runs over the primes dividing the order of $G$, and $W_{p}$ is the subgroup generated by the $p$-Sylow subgroups of $G$. Ribenboim proved that any finite group is the active sum of the family $\mathcal{F}$ of the subgroups of $G$ of prime power order with $\mathcal{F}$ ordered by inclusion. A. Díaz-Barriga and L. Román obtained a family (also ordered by inclusion) more economical than $\mathcal{F}$ whose active sum is $G$, namely the family of tame intersections of subgroups of prime power order.

Perhaps another motivating question for the concept of active sum, related to the original one, is the following: Is there an analogue for (not necesarily abelian) groups of the theorem stating that any finitely generated abelian group is a direct sum of cyclic subgroups?

The concept of active sum is proposed as an analogue for groups of the concept of direct sum for abelian groups (in fact Tomàs sometimes uses the term direct sum for this construction).

Should the 'building blocks' be cyclic groups? Ribenboim proposes the atomic subgroups (also called groups of weight at most one [24]) as building blocks. These are the groups (important in knot theory) normally generated by one element. It is known (see [26], [27]) that the finite atomic groups are the finite groups with cyclic abelianization. 
Ribenboim defines a molecular group as one which is an active sum of atomic groups.

Non-molecular groups are not exhibited in Ribenboim's paper. Tomás and Belana [3] have constructed a 2-group which is not an active sum of any discrete family of proper normal subgroups. We defer to the sequel of this paper the proof that it is not molecular. Two intriguing questions are: which finite groups are molecular?; which finite groups are active sums of cyclic subgroups? We will present in the sequel of this paper families of molecular and non-molecular groups.

Besides the applications in Galois groups and finite group theory that appear in [43], [42], [36], [7], [6], [34], active sums (of groups) appear in the study of subgroups of amalgamated free products (see [30] and Section 2.2.2), knot groups (see [39] and Section 2.2.5), and Coxeter groups (see [22] and Section 2.2.4). Other articles dealing with the active sum are [8], [32], [33]

Lyndon and Schupp in [30], Section I.11, consider a free product with amalgamation, $G=*_{A} H_{i}$, and describe what in our terminology is an active family of subgroups of $G$. They prove (in Proposition 11.4) that the active sum of this family is $G$, though the proof is marred with many errors; use of our Corollary 2.5 provides an easy conceptual proof. More generally, for any subgroup $G^{*}$ of $G$, they describe an active family of subgroups of $G^{*}$, with active sum $N$, a normal subgroup of $G^{*}$ such that $G^{*} / N$ is free.

Simon [39] considers finitely presented groups $G$ with infinite cyclic abelianization and normally generated by one element $t$, and takes the active sum $S$ of the family of conjugates of the subgroup generated by $t$. He called $S$ a best Wirtinger approximation of $G$; the group $G$ is the fundamental group of a closed orientable surface in $\mathbb{R}^{4}$ if and only if $S$ is isomorphic to $G$ for some suitable choice of $t$.

Here is an example considered in [16] and [13], which will not be pursued in this paper. Let $M_{g}$ be the mapping class group of the closed orientable surface $F_{g}$ of genus $g$, that is, the quotient of the group of orientation-preserving homeomorphisms of $F_{g}$ by the subgroup of homeomorphisms isotopic to the identity. Let $t$ be a Dehn twist along a non-separating curve, that is, an element of $M_{g}$ which is not a proper power and is represented by a homeomorphism of $F_{g}$ which is the identity in the complement of a non-separating annulus. All such $t$ are conjugate up to inversion. By [28] $t$ normally generates $M_{g}$. Again, consider the active sum $S_{g}$ of the family of conjugates of the subgroup generated by $t$. When $g \geq 3$ it turns out that $H_{1}\left(M_{g}\right)=0$, $H_{2}\left(M_{g}\right)=\mathbb{Z}[16], H_{1}\left(S_{g}\right)=\mathbb{Z}$, and the (central) kernel of the natural epimorphism from $S_{g}$ onto $M_{g}$ is $\mathbb{Z}^{2}$, that is, $S_{g}$ is the direct product of $\mathbb{Z}$ with the universal central extension of $M_{g}$. This example is important in the obtention of a finite presentation of $M_{g}[17,45]$, and in the proof of Kirby's surgery theorem [25,29]. A related example with Dehn twists along all curves is considered in [13].

In Section 2.2.4, we show that any Coxeter group is the active sum of a family of subgroups of order 2. A 3-transposition group $G$ [1] yields an additional example of a generating active family of subgroups of order two. In this case two different 
subgroups of the family generate a subgroup of order at most six.

The concept of active sum, or that of active add, is a common, though not always explicit, ingredient in many seemingly unrelated papers, some of them with topological applications. Aside from those mentioned above, we mention [10], [40], and [24].

The paper is organized as follows. In Section 1, we give a construction of the active sum of groups using partial algebras (see [5]). We find necessary and sufficient conditions on a generating active family $\mathcal{F}$ of subgroups of a group $G$, for the abelianization of the active sum of $\mathcal{F}$ to be isomorphic to the abelianization of $G$. The conditions found here are called regularity and independence.

In Section 2 we observe that it suffices to split the canonical epimorphism from the active sum to the group in question, to prove that they are isomorphic, provided that the family under consideration is regular and independent. We use this result in a few examples: free groups, free amalgamated products, semidirect products of cyclic groups, Coxeter groups, and best Wirtinger approximations. We also introduce atomic and molecular groups, showing that a finite class 2 group is molecular if and only if it is the active sum of cyclic subgroups. In Section 2.4 we show that the pullback of an active sum of groups is the active sum of the inverse image of the active family of the original group. We use this result to show that every finite, non-cyclic group is the active sum of proper normal subgroups, and that every finite group that is not a cyclic $p$-group is the active sum of proper subgroups. We conclude the section with the study of the active sum via homology. We show here, that, if the Ganea map [12] in certain exact sequence is an epimorphism, then regularity and independence of the generating active family of subgroups suffice for the active sum to be isomorphic to the group $G$ in question. In particular, the result applies when the second homology group of $G$ is trivial. A less trivial corollary of the aforementioned result is used to show that any group of order $p^{3}$ with $p$ an odd prime, is the active sum of cyclic subgroups.

In section 3 we point out that independence is automatic for perfect groups. We deal next with some examples of perfect groups, and some examples of simple groups. We show that every finite simple group with trivial Schur multiplier is the active sum of cyclic subgroups. We investigate the finite special linear groups $\mathrm{SL}_{n}(q)$. When $n \geq 3$, previous results show that $\mathrm{SL}_{n}(q)$ is the active sum of cyclic subgroups, with a few exceptions. The analysis for the case $n=2$ involves direct computations. The main result of section 3.4 is that, for a finite group $G$, and any generating regular and independent active family $\mathcal{F}$ of subgroups of $G$, if the order of any element of $\mathcal{F}$ is relatively prime to the order of the second homology group of $G$ then the active sum of $\mathcal{F}$ is a covering group of $G$.

Some of the experimental evidence for this paper was gathered using the computer programs Maple and Gap. 


\section{The active sum of groups}

\subsection{The active sum via partial algebras}

We give a construction of the active sum of groups using partial algebras. This construction is economical notationally and conceptually. For the definition and properties of partial algebras we refer the reader to [5]. We start by defining the categories Add and Actad. In the terminology of [5], Add is the category of partial algebras with one operation ' ' of arity two; and Actad is the category of partial algebras with two operations ' $'$ ', and ' $\wedge$ ', both with arity two. What this means is that an object of the category Add, called an add, consists of a set $A$, together with a partial binary operation:

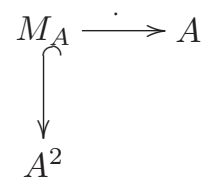

called multiplication. A morphism of adds $f: A \rightarrow B$ is a function $f: A \rightarrow B$, such that $f \times f\left(M_{A}\right) \subseteq M_{B}$, and the diagram

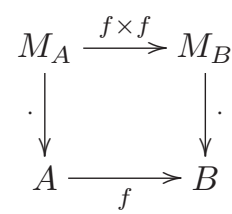

commutes. Composition is composition of functions. An object $A$ in the category Actad, also called an active add, consists of a set $A$, together with two partial binary functions
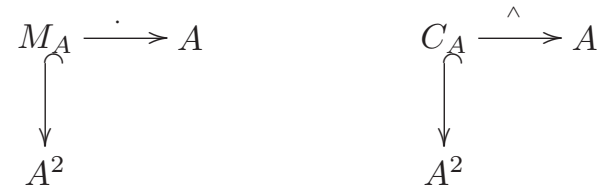

that we call multiplication and conjugation. A morphism $f: A \rightarrow B$ between active adds is a function $f: A \rightarrow B$ such that $f \times f\left(M_{A}\right) \subseteq M_{B}$ and $f \times f\left(C_{A}\right) \subseteq C_{B}$, and the diagrams
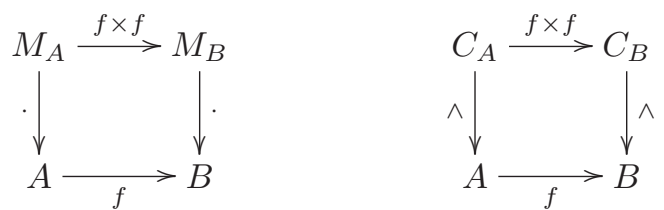

commute. Given $a, b \in A$, we write $\cdot(a, b)=a \cdot b$ and ${ }^{\wedge}(a, b)=a^{b}$. Adds can be traced back to [2]. 
Denote the category of groups and homomorphisms by Grp. We have a full and faithful functor $F:$ Grp $\rightarrow$ Actad defined as follows: Given a group $G$, the active add $F(G)$ has the same underlying set as $G, M_{F(G)}=C_{F(G)}=G^{2}$; the function $M_{F(G)} \rightarrow G$ is multiplication in the group $G$, and the function $C_{F(G)} \rightarrow G$ is such that $(h, g) \mapsto g^{-1} h g$. If we keep only the first partial function we obtain a functor Grp $\rightarrow$ Add

[5] gives an explicit construction of the product in a category of partial algebras of a given similarity type. It is not hard to see that this category also has equalizers of pairs of arrows: given $f, g: A \rightarrow B$ the set $\{a \in A \mid f(a)=g(a)\}$ is a closed subset of the partial algebra $A$, and it determines a closed subalgebra that is the equalizer of $f$ and $g$. What this means is that the category of partial algebras of a given similarity type is complete [31]. Analyzing these constructions in the case of Actad, it is not hard to see that the functor $F:$ Grp $\rightarrow$ Actad defined above preserves limits.

Proposition 1.1. The functor $F:$ Grp $\rightarrow$ Actad has a left adjoint.

Proof. Consider the following commutative diagram:

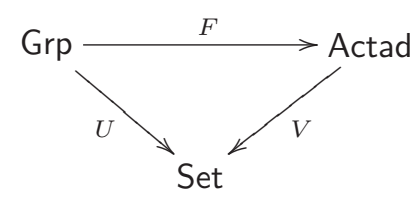

where Set is the category of sets and $U$ and $V$ are forgetful functors. As we mentioned before, $F$ preserves limits. It is well known that the functor $U$ has a left adjoint. $V$ is clearly faithful. Furthermore, Grp is complete, well powered and well co-powered. These conditions suffice for the existence of a left adjoint to $F$ [18], pg. 213.

Denote the left adjoint of $F$ by $S:$ Actad $\rightarrow$ Grp. Explicitly, given an active add $A$, we have that $S(A)$ is the free group generated by $A$ modulo the normal subgroup generated by elements of the form

$$
\begin{gathered}
a b(a \cdot b)^{-1} \quad \text { with }(a, b) \in M_{A} \\
b^{-1} a b\left(a^{b}\right)^{-1} \quad \text { with }(a, b) \in C_{A} .
\end{gathered}
$$

Explicit constructions for coproducts and directed colimits in the category of partial algebras for a given similarity type are also given in [5]. Furthermore, the analysis given there of congruences makes it clear that coequalizers also exist: Given $f, g: A \rightarrow B$, consider the smallest congruence $\theta$ on $B$ that contains the pairs $(f(a), g(a))$ for all $a \in A$, then the coequalizer is the quotient morphism $B \rightarrow B / \theta$. This means that the category of partial algebras for a given similarity type is cocomplete.

As examples, if $R$ is a rack where we view $R$ as an active add with empty domain of the multiplication and global conjugation, then $S(R)$ is the associated group defined 
in [10]; if $R$ is a pregroup [40], chapter 3, viewed as an active add where the domain for the conjugation is empty, then $S(R)$ is the universal group of $R$.

Definition 1.2. (i) An active family of adds consists of a functor $\Gamma: \mathbf{I} \rightarrow$ Add, where $\mathbf{I}$ is a small category, together with an active add $A$ whose underlying add is $\lim _{I \in \mathbf{I}} \Gamma I$.

(ii) We call $S(A)$ the active sum of the active family of adds.

The situation we consider in this paper is as follows. Let $G$ be a group and $\mathbf{I}$ a partially ordered set. Denote by $\operatorname{Sub}(G)$ the partially ordered set of subgroups of $G$, where the order is given by inclusion. Consider an injective, order preserving map $\mathbf{I} \rightarrow \operatorname{Sub}(G)$, and denote by $\Gamma$ the functor

$$
\mathbf{I} \rightarrow \operatorname{Sub}(G) \rightarrow \operatorname{Grp} \rightarrow \operatorname{Add} .
$$

We then have the add $\lim _{\longrightarrow} \Gamma$ (the colimit is taken in the category of adds). To use the conjugation in the group $G$ to define a total operation $\left(\lim _{\mathbf{I}} \Gamma\right)^{2} \rightarrow \lim _{\mathbf{I}} \Gamma$, making it an object of Actad, we would need the family $\langle\Gamma I\rangle_{I \in \mathbf{I}}$ to satisfy the following conditions:

(a) It is closed under conjugation (i.e. for every $I, J \in \mathbf{I}$ and $g \in \Gamma J$, there exists a $K \in \mathbf{I}$ such that $\left.\Gamma K=\Gamma I^{g}\right)$.

(b) The order in $\mathbf{I}$ is compatible with conjugation (i.e. If $I, I^{\prime}, J, J^{\prime}, K \in \mathbf{I}$ with $I \leq J$, and $g \in \Gamma K$ is such that $\Gamma{I^{\prime}}^{g}=\Gamma I$ and $\Gamma J^{\prime g}=\Gamma J$, then $I^{\prime} \leq J^{\prime}$ in $\mathbf{I}$.

More generally, we have

Lemma 1.3. Let $C$ be an active add where the operation $\wedge: C \times C \rightarrow C$ is total. Let $S \subseteq C^{2}$ and $\theta$ be the congruence generated by $S$ in the underlying add of $C$. Then $\theta$ is a congruence in the category Actad, if the following conditions are satisfied for all $a, b, h, k \in C$ :

(i) If $(a, b) \in S$, then $\left(a^{h}, b^{h}\right) \in \theta$.

(ii) If $(h, k) \in S$, then $\left(a^{h}, a^{k}\right) \in \theta$.

(iii) $\left((a \cdot b)^{h}, a^{h} \cdot b^{h}\right) \in \theta$ if $a \cdot b$ is defined in $C$.

(iv) $\left(a^{h \cdot k},\left(a^{h}\right)^{k}\right) \in \theta$ if $h \cdot k$ is defined in $C$.

Proof. For every $h \in C$ define $\varphi_{h}: C \rightarrow C / \theta$ such that $\varphi_{h}(a)=\left[a^{h}\right]$, for every $a \in C$. Using condition (iii), it is not hard to see that $\varphi_{h}$ is a morphism of adds. Condition (i) tells us that $S \subseteq \operatorname{ker} \varphi_{h}$, where $\operatorname{ker} \varphi_{h}=\left\{(a, b) \in C^{2} \mid \varphi_{h}(a)=\varphi_{h}(b)\right\}$. From [5] we know that $\operatorname{ker} \varphi_{h}$ is a congruence. Therefore $\theta \subseteq \operatorname{ker} \varphi_{h}$. That is to say, for every $(a, b) \in \theta$ we have $\left(a^{h}, b^{h}\right) \in \theta$. Similarly, for every $a \in C$, we define $\psi_{a}: C \rightarrow C / \theta$ such that for every $h \in C$ we have $\psi_{a}(h)=\left[a^{h}\right]$. We have then that $\theta \subseteq \operatorname{ker} \varphi_{a}$. Therefore, for every $(h, k) \in \theta$ we have that $\left(a^{h}, a^{k}\right) \in \theta$. We thus conclude that for every $(a, b),(h, k) \in \theta$ we have $\left(a^{h}, b^{k}\right) \in \theta$. 
The situation we were talking about before the lemma corresponds to $C=\coprod_{I \in \mathbf{I}} \Gamma I$, and $\theta$ the congruence of adds such that $C / \theta=\lim _{I \in \mathbf{I}} \Gamma I$.

It is worth mentioning that the definition of active quiver of groups given in [35] produces an active add in our context, and the active sum, in the sense of [35], of the active quiver and the left adjoint $S$ given here applied to the active add are isomorphic.

\subsection{Regularity and independence}

Let $G$ be a group and let $\mathcal{F}=\left\langle F_{i}\right\rangle_{i \in I}$ be a family of pairwise different subgroups of $G$ closed under conjugation. What we mean by closed under conjugation is, that for every element $F_{i} \in \mathcal{F}$ and every element $g \in G$ we have that $F_{i}^{g} \in \mathcal{F}$, where $F_{i}^{g}=g^{-1} F_{i} g$. For $h \in F_{i}$ we denote the element $g^{-1} h g \in F_{i}^{g}$ by $h^{g}$. Assume we have a partial order $\leq$ on $\mathcal{F}$ compatible with group inclusion and conjugation. That is to say, for all $i, j \in I$ we have that $F_{i} \leq F_{j}$ implies that $F_{i}$ is a subgroup of $F_{j}$, and that for all $k \in F_{\ell} \in \mathcal{F}$ we have that $F_{i}^{k} \leq F_{j}^{k}$. A family $\mathcal{F}$ with these properties will be called an active family of subgroups of $G$. If the family generates the group $G$, we will call it a generating active family. When the order is the trivial one we say that the family $\mathcal{F}$ is discrete.

Such a family $(\mathcal{F}, \leq)$ is an active family in the sense of [35]. Therefore we can consider its active sum $S$. Explicitly, we have that $S=\star \mathcal{F} / R$, where $\nsucc \mathcal{F}$ is the free product of the elements of $\mathcal{F}$, and $R$ is the normal subgroup of $\star \mathcal{F}$ generated by elements of the form:

(i) $h^{-1} g h\left(g^{h}\right)^{-1}$ with $g \in F_{i}$ and $h \in F_{j}$.

(ii) $g c_{i j}(g)^{-1}$ with $g \in F_{i}, F_{i} \leq F_{j}$ in $\mathcal{F}$ and $c_{i j}: F_{i} \rightarrow F_{j}$ the inclusion map.

By the universal property of the active sum, there exists a unique homomorphism $\varphi: S \rightarrow G$ such that for every $i \in I$ the diagram

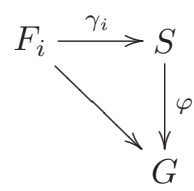

commutes, where $\gamma_{i}$ is the canonical injection of $F_{i}$ into the active sum. If the family $\mathcal{F}$ generates $G$ then $\varphi: S \rightarrow G$ is an epimorphism.

Remark 1.4. It is of course possible to consider a more general definition allowing for the family of subgroups to have repetitions by introducing a function

$$
k: I \times\left(\coprod_{i} F_{i}\right) \rightarrow I
$$

such that $F_{k(i, y)}=y^{-1} F_{i} y$. 
We assume for the rest of the section that $\mathcal{F}$ is a generating active family of subgroups of $G$. We will make use of homology theory; the following lemma will allow us to do so.

Lemma 1.5. If $Z(S)$ is the center of $S$ and $Z(G)$ is the center of $G$, then $\varphi^{-1}(Z(G))=$ $Z(S)$.

Proof. Since $\varphi$ is an epimorphism, $Z(S) \subseteq \varphi^{-1}(Z(G))$. For the converse, consider an element of $S$ represented by $g \in F_{k}$ and let $w \in S$. We can write $w=f_{1} f_{2} \cdots f_{n}$ where $f_{i} \in F_{j_{i}}(i=1, \ldots, n)$. It is easy to show, by induction on $n$, that the element $w^{-1} g w$ of $S$ is represented by the element $\varphi(w)^{-1} g \varphi(w)$ of $F_{k}^{\varphi(w)}$. In particular, if $\varphi(w) \in Z(G)$, then $w^{-1} g w=g$ for any $g \in F_{k}$ and any $k$, and therefore $w \in Z(S)$.

Consider the following short exact sequence

$$
1 \longrightarrow \operatorname{ker}(\varphi) \longrightarrow S \stackrel{\varphi}{\longrightarrow} G \longrightarrow 1
$$

As a consequence of the previous lemma, we have that $\operatorname{ker}(\varphi) \subseteq Z(S)$. Therefore ([21], pg. 203) we have an exact sequence

$$
H_{2}(S) \stackrel{\varphi_{*}}{\longrightarrow} H_{2}(G) \longrightarrow \operatorname{ker}(\varphi) \longrightarrow H_{1}(S) \stackrel{\varphi_{*}}{\longrightarrow} H_{1}(G) \longrightarrow 0 \text {. }
$$

We have that $\varphi$ is an isomorphism if and only if $\varphi_{*}$ is a monomorphism in $H_{1}$ (and thus, an isomorphism), and an epimorphism in $H_{2}$. We will find necessary and sufficient conditions on the family $\mathcal{F}$ for $\varphi_{*}: H_{1}(S) \rightarrow H_{1}(G)$ to be an isomorphism.

Define the following graph $\mathbf{I}$. The objects of $\mathbf{I}$ are the elements of $I$. There is in $\mathbf{I}$ at most one arrow from one object to another. There is an arrow $i \rightarrow j$ in $\mathbf{I}$ if either of the following two conditions is satisfied:

(i) $F_{i} \leq F_{j}$ in $\mathcal{F}$.

(ii) There is a $k \in I$ and a $g \in F_{k}$ such that $F_{j}=F_{i}^{g}$.

Consider the following diagram $\Gamma: \mathbf{I} \rightarrow$ Grp: For every $i \in I$ define $\Gamma(i)=F_{i} / R_{i}$, where $R_{i}$ is the normal subgroup of $F_{i}$ generated by elements of the form $x^{-1} x^{g}$, where $x \in F_{j}, g \in G$, and $F_{j} \leq F_{i} \geq F_{j}^{g}$ in $\mathcal{F}$. If the arrow $i \rightarrow j$ comes from the condition $F_{i} \leq F_{j}$ then, define $\Gamma(i \rightarrow j)$ as the homomorphism $F_{i} / R_{i} \rightarrow F_{j} / R_{j}$ induced by the inclusion $F_{i} \rightarrow F_{j}$. If, on the other hand, we have that the arrow $i \rightarrow j$ in $\mathbf{I}$ comes from $F_{j}=F_{i}^{g}$, then define $\Gamma(i \rightarrow j)$ as the homomorphism $F_{i} / R_{i} \rightarrow F_{j} / R_{j}$ induced by the homomorphism $x \mapsto g^{-1} x g$ from $F_{i}$ to $F_{j}$. It is not hard to see that the definition of $\Gamma$ does not depend on the choice of $g$ such that $F_{j}=F_{i}^{g}$.

Proposition 1.6. With the above notation, we have that $S / S^{\prime} \simeq \lim _{\mathbf{I}} F_{i} / R_{i}$, where the colimit is taken in the category of abelian groups. 
Proof. It is not hard to see that $R_{i}$ is contained in the kernel of the composition

$$
F_{i} \rightarrow S \rightarrow S / S^{\prime}
$$

where the first map is the canonical inclusion of $F_{i}$ in the active sum $S$, and the second is the projection of $S$ onto its abelianization. Therefore we have a map $F_{i} / R_{i} \rightarrow S / S^{\prime}$. For every $i \rightarrow j$ in $\mathbf{I}$ we have that the diagram

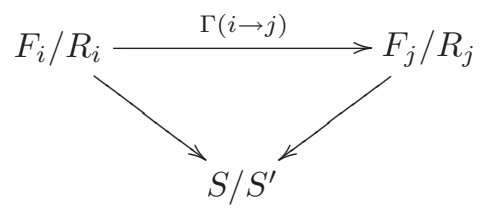

commutes. There exists then a unique homomorphism $\lim _{\mathbf{I}} F_{i} / R_{i} \rightarrow S / S^{\prime}$ such that the diagram

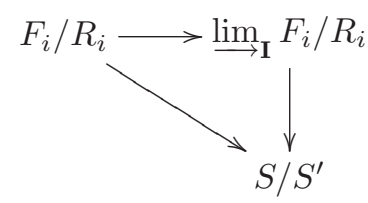

commutes for every $i \in I$.

On the other hand, the family of homomorphisms

$$
F_{i} \rightarrow F_{i} / R_{i} \rightarrow \underset{\mathbf{I}}{\lim } F_{i} / R_{i}
$$

indexed by $I$ satisfies the appropriate conditions to apply the universal property of the active sum $S$. Therefore, we have a unique homomorphism $S \rightarrow \lim _{\longrightarrow} F_{i} / R_{i}$ such that the diagram

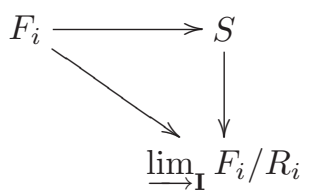

commutes. Since $\lim _{\mathbf{I}} F_{i} / R_{i}$ is abelian, we have an induced homomorphism $S / S^{\prime} \rightarrow$ $\lim _{\mathbf{I}} F_{i} / R_{i}$.

The two morphisms thus induced between $S / S^{\prime}$ and $\lim _{\mathbf{I}} F_{i} / R_{i}$ are inverses of each other.

Observe that we have, for every $i \in I$, the following short exact sequence of abelian groups

$$
0 \longrightarrow \frac{F_{i} \cap G^{\prime}}{R_{i}} \stackrel{\eta_{i}}{\longrightarrow} \frac{F_{i}}{R_{i}} \stackrel{\psi_{i}}{\longrightarrow} \frac{F_{i} G^{\prime}}{G^{\prime}} \longrightarrow 0
$$


where $\eta_{i}$ is the inclusion. We obtain the following commutative diagram of abelian groups with exact rows [38], pp. 46-47:

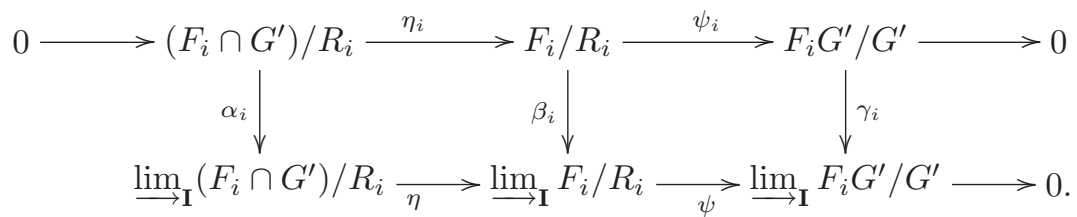

In this context, we give the following definition:

Definition 1.7. We say that $\mathcal{F}$ is regular if $\beta_{i}\left(\left(F_{i} \cap G^{\prime}\right) / R_{i}\right)=0$ for every $i \in I$.

Next theorem follows immediately from the definition:

Theorem 1.8. The following statements are equivalent.

(i) $\mathcal{F}$ is regular.

(ii) $\eta\left(\lim _{\mathbf{I}}\left(F_{i} \cap G^{\prime}\right) / R_{i}\right)=0$

(iii) $\psi$ is an isomorphism.

We have the following result for discrete families.

Lemma 1.9. Assume that the family $\mathcal{F}$ is discrete. The family $\mathcal{F}$ is regular if and only if $\left[F, N_{G}(F)\right]=F \cap G^{\prime}$ for every $F \in \mathcal{F}$, where $N_{G}(F)$ is the normalizer of $F$ in $G$.

Proof. If the family $\mathcal{F}$ is discrete, then $R_{i}=\left[F_{i}, N_{G}\left(F_{i}\right)\right]$ for every $i$. Then clearly $F_{i} \cap G^{\prime} / R_{i}=0$. Therefore $\mathcal{F}$ is regular. Suppose now that $\mathcal{F}$ is regular. Then, two elements $i, j$ in the same connected component of the diagram I satisfy $i \leq j$ and $j \leq i$. The induced morphism $F_{i} / R_{i} \rightarrow F_{j} / R_{j}$ is an isomorphism. If we take one representative in every connected component of $\mathbf{I}$, and call $T$ the set of representatives, we have that $\lim _{\longrightarrow} F_{i} / R_{i} \simeq \bigoplus_{t \in T} F_{t} / R_{t}$. Given $i$, we may assume that $i \in T$. Then $\beta_{i}$ is basically the coprojection $F_{i} / R_{i} \rightarrow \bigoplus_{t \in T} F_{t} / R_{t}$. Since $\mathcal{F}$ is regular, we have that $F_{i} \cap G^{\prime} \subseteq R_{i}=\left[F_{i}, N_{G}\left(F_{i}\right)\right]$. The inclusion $\left[F_{i}, N_{G}\left(F_{i}\right)\right] \subseteq F_{i} \cap G^{\prime}$ is always satisfied.

In some cases it is easier to work with what we call a transversal. We define the equivalence relation $\sim$ on $I$ as follows. For $i, j \in I$ we say that $i \sim j$ if and only if there is a $g \in G$ such that $F_{i}^{g}=F_{j}$. A subset $T \subseteq I$ that contains exactly one representative for each class of equivalence of $\sim$ is called a transversal. It is not hard to see that

Lemma 1.10. $\mathcal{F}$ is regular if for every $t \in T$, there exists an $i \in I$ with $i \sim t$, such that $\beta_{i}\left(\left(F_{i} \cap G^{\prime}\right) / R_{i}\right)=0$. 
We define the graph $\mathbf{T}$ from $T$ and $\sim$ as follows: The objects of $\mathbf{T}$ are the elements of $T$. There is at most one arrow in $\mathbf{T}$ between any two objects $s$ and $t$ in $\mathbf{T}$. There is an arrow $s \rightarrow t$ in $\mathbf{T}$ if we can find $i \sim s$ and $j \sim t$ such that there is an arrow $i \rightarrow j$ in $\mathbf{I}$.

We now define a diagram from $\mathbf{T}$ to the category of abelian groups: for every $t \in \mathbf{T}$ let $A_{t}=F_{t} G^{\prime} / G^{\prime}$. If there is an arrow $s \rightarrow t$ in $\mathbf{T}$, then we can induce a canonical homomorphism $A_{s} \rightarrow A_{t}$. We can then consider the abelian group $\lim _{\longrightarrow} A_{t}$.

For every $i \in I$ there is a $t \in T$ such that $i \sim t$. We therefore have an isomorphism $F_{i} G^{\prime} / G^{\prime} \rightarrow A_{t}$. These isomorphisms induce a homomorphism

$$
\underset{\mathbf{I}}{\lim _{i}} F_{i} G^{\prime} / G^{\prime} \rightarrow \underset{\mathbf{T}}{\lim } A_{t}
$$

It is not hard to see that the above homomorphism is an isomorphism.

Now, for every $t \in \mathbf{T}$ we have an obvious monomorphism $A_{t} \rightarrow G / G^{\prime}$. Furthermore, if $s \rightarrow t$ in $\mathbf{T}$, the diagram

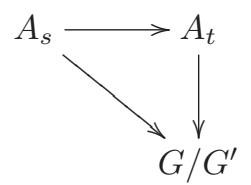

commutes. We can induce then a unique homomorphism $\varepsilon: \lim _{\mathbf{T}} A_{t} \rightarrow G / G^{\prime}$ such that for every $t \in \mathbf{T}$ the diagram

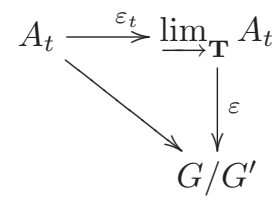

commutes, where $\varepsilon_{t}$ is the canonical homomorphism into the colimit. Since the homomorphism $A_{t} \rightarrow G / G^{\prime}$ is mono, we conclude that every $\varepsilon_{t}$ is also mono. Observe furthermore that $\varepsilon$ is an epimorphism.

Definition 1.11. $\mathcal{F}$ is called independent if the homomorphism $\varepsilon: \lim _{\mathbf{T}} A_{t} \rightarrow G / G^{\prime}$ is an isomorphism.

Theorem 1.12. $\varphi_{*}: S / S^{\prime} \rightarrow G / G^{\prime}$ is an isomorphism if and only if $\mathcal{F}$ is regular and independent.

Proof. The composition

$$
S / S^{\prime} \longrightarrow \lim _{\mathbf{I}} F_{i} / R_{i} \longrightarrow \lim _{\mathbf{I}} F_{i} G^{\prime} / G^{\prime} \longrightarrow \lim _{\mathbf{T}} A_{t} \longrightarrow G / G^{\prime}
$$

is the homomorphism $\varphi_{*}: S / S^{\prime} \rightarrow G / G^{\prime}$ induced by $\varphi: S \rightarrow G$. 


\section{First conditions for recovering a group as an active sum}

\subsection{Generating sets for the active sum}

We show in this section that, for any regular and independent family of subgroups of a group $G$, a subset of the active sum generates the active sum if its image under the canonical projection to $G$ generates $G$. An interesting corollary in such a case is that, to prove that the active sum is isomorphic to the given group, it suffices to split the canonical projection from the active sum to the group.

Lemma 2.1. Let $H$ be a subgroup of a group $G$. If $H G^{\prime}=G$ and $H Z(G)=G$ then $H=G$

Proof. If $H Z(G)=G$, we have that for every $g_{1}, g_{2} \in G$, we can find $h_{1}, h_{2} \in H$ and $z_{1}, z_{2} \in Z(G)$ such that $g_{i}=h_{i} z_{i}$. Clearly $\left[g_{1}, g_{2}\right]=\left[h_{1} z_{1}, h_{2} z_{2}\right]=\left[h_{1}, h_{2}\right]$. Therefore $G^{\prime}=H^{\prime}$. Then $G=H G^{\prime}=H H^{\prime}=H$.

Corollary 2.2. Let $G$ be a group and $X \subset G$ a subset. If the images of $X$ under the canonical homomorphisms $G \rightarrow G / G^{\prime}$, and $G \rightarrow G / Z(G)$ generate $G / G^{\prime}$ and $G / Z(G)$ respectively, then $X$ generates $G$.

Corollary 2.3. If $\varphi: S \rightarrow G$ is an epimorphism of groups, such that $\operatorname{ker} \varphi \subseteq$ $S^{\prime} \cap Z(S)$, then a subset $X$ of $S$ generates $S$ if and only if $\varphi(X)$ generates $G$.

Proof. Assume that $\varphi(X)$ generates $G$. Consider

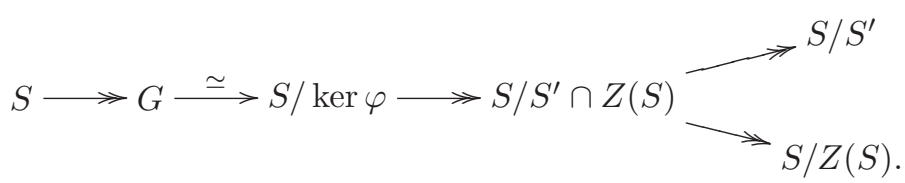

Observe that every arrow is an epimorphism. Since $\varphi(X)$ generates $G$, we have that its images under $S \rightarrow S / S^{\prime}$ and $S \rightarrow S / Z(S)$ generate $S / S^{\prime}$ and $S / Z(S)$ respectively. According to the previous corollary, we have that $X$ generates $S$.

We can now show:

Proposition 2.4. Let $G$ be a group, and let $\mathcal{F}$ be a generating active family of subgroups of $G$. Let $S$ be the active sum of the family $\mathcal{F}$, and let $\varphi: S \rightarrow G$ be the canonical homomorphism. If $\mathcal{F}$ is regular and independent, then a subset $X \subseteq S$ generates $S$ if and only if $\varphi(X)$ generates $G$.

Proof. The fact that $\mathcal{F}$ is regular and independent means that the bottom arrow of the commutative diagram

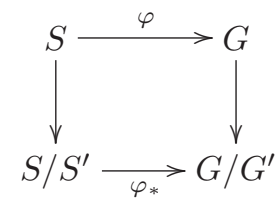


is an isomorphism. It is easy to see then, that $\operatorname{ker} \varphi \subseteq S^{\prime}$. On the other hand, according to Lemma 1.1.5, $\operatorname{ker} \varphi \subseteq Z(S)$. Now apply the previous corollary.

Corollary 2.5. Let $\mathcal{F}$ be a regular and independent generating active family of subgroups of a group $G$. Let $S$ be the active sum of the family $\mathcal{F}$, and $\varphi: S \rightarrow G$ the canonical projection. The epimorphism $\varphi: S \rightarrow G$ splits if and only if $\varphi$ is an isomorphism.

\subsection{Examples}

We use the above results to prove that several groups are active sums of certain families of subgroups, cyclic subgroups in most cases.

\subsubsection{FREE GROUPS}

As a first example, let $F$ be a free group on the set $X$. Let $\mathcal{F}:=\left\{\left\langle x^{w}\right\rangle \mid x \in X, w \in\right.$ $F\}$. It is clear that $\mathcal{F}$ is a generating active family of cyclic subgroups of $F$.

Since $\left\langle x^{w}\right\rangle \cap F^{\prime}$ is trivial for every element of $\mathcal{F}$, we have that the family is regular.

It is also easy to see that we can choose the family $\{\langle x\rangle\}_{x \in X}$ as representative groups for the transversal. Then the colimit $\lim _{x \in X}\langle x\rangle F^{\prime} / F^{\prime}$ in the category of abelian groups is isomorphic to the free abelian group in $X$, and isomorphic to it by the homomorphism induced by the inclusions $\langle x\rangle F^{\prime} / F^{\prime} \rightarrow F / F^{\prime}$. Thus the family is independent.

Let $S$ be the active sum of the family $\mathcal{F}$. According to Corollary 2.5, to show that $S$ is isomorphic to $F$ it suffices to split the canonical projection $\pi: S \rightarrow F$. This is easily done since $F$ is free.

\subsubsection{Free Amalgamated products}

Let $\left\langle H_{\nu}\right\rangle_{\nu \in N}$ be a family of groups and $A$ another group. Assume that, for every $\nu \in N$ we have an injective homomorphism $\varphi_{\nu}: A \rightarrow H_{\nu}$ that is not an isomorphism. Let $G$ be the amalgamated product of the groups $H_{\nu}$ with amalgamated subgroup $A$. For details of this construction we refer the reader to [30]. Let $\psi_{\nu}: H_{\nu} \rightarrow G$ be the usual homomorphism. It is well known that $\psi_{\nu}$ is an injection, so that we can identify $H_{\nu}$ with a subgroup of $G$. It is also well known that $G$ is the colimit in Grp of the diagram

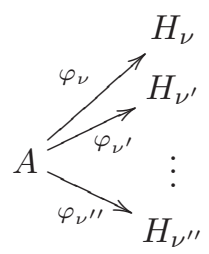

with injections $\psi_{\nu}: H_{\nu} \rightarrow G$. 
We want to express $G$ as the active sum of the active family of subgroups formed by all the conjugates of $A$ in $G$ plus all the conjugates of $H_{\nu}$ in $G$, for all $\nu \in N$, ordered by inclusion. We denote by $S$ the active sum of this family, and let $\varphi: S \rightarrow G$ be the usual projection. Observe that the diagram

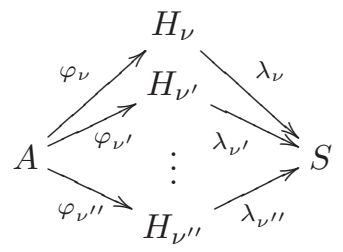

commutes, where $\lambda_{\nu}: H_{\nu} \rightarrow S$ is the usual homomorphism to the active sum. By the universal property of the colimit, there is a unique homomorphism $\pi: G \rightarrow S$ such that $\pi \circ \psi_{\nu}=\lambda_{\nu}$ for every $\nu \in N$. It is clear that the diagram

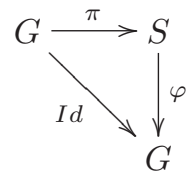

commutes. By Corollary 2.5, $\varphi$ is an isomorphism.

As mentioned in the introduction, [30] also considers a subgroup $G^{*}$ of $G$, and constructs an active family of subgroups of $G^{*}$ whose active sum is a normal subgroup $N$ of $G^{*}$ such that $G^{*} / N$ is free. In this case, as in the previous one, the families considered allow repetitions of the subgroups, see Remark 1.1.4.

\subsubsection{SEmidireCt PROdUCTS OF CYCLIC GROUPS}

We show now that the semidirect product $G:=\mathbb{Z}_{s} \propto^{t} \mathbb{Z}_{r}$ of $\mathbb{Z}_{s}$ and $\mathbb{Z}_{r}$ by $t$ can be expressed as an active sum of cyclic subgroups. We recall that $G$ has two generators $x$ and $y$ with the following properties: $x^{r}=y^{s}=1$ and $y^{-1} x y=x^{t}$. Since $x$ has order $r$, we have $t^{s} \equiv 1 \bmod r$. This in particular means that $(r, t)=1$.

Observe that the conjugates of $y$ are $y, y x^{t-1}, y x^{2(t-1)}, \ldots, y x^{(r-1)(t-1)}$. Let $F_{i}=\left\langle y x^{i(t-1)}\right\rangle$ for $i=1,2, \ldots, r$, and let $F_{0}=\langle x\rangle$. Consider the discrete generating active family $\mathcal{F}=\left\langle F_{i}\right\rangle_{i=0}^{r}$. Let us see that $\mathcal{F}$ is regular and independent.

It is not hard to see that $G^{\prime}=\left\langle x^{t-1}\right\rangle$. Also, $R_{0}=\left\langle x^{t-1}\right\rangle$. Thus $\left(F_{0} \cap G^{\prime}\right) / R_{0}$ is trivial. It can be shown that

$$
\left(y x^{i(t-1)}\right)^{m}=y^{m} x^{i\left(t^{m}-1\right)} .
$$

In particular $\left(y x^{i(t-1)}\right)^{s}=y^{s} x^{i\left(t^{s}-1\right)}=1$. We conclude that $F_{i} \cap G^{\prime}$ is trivial if $i \neq 0$. Therefore $\mathcal{F}$ is regular.

Now we see that $\mathcal{F}$ is independent. As representatives for the transversal we can consider $F_{0}$ and $F_{1}$. Observe that $F_{0} G^{\prime} / G^{\prime} \simeq\langle x\rangle /\left\langle x^{t-1}\right\rangle$. On the other hand 
$F_{1} G^{\prime} / G^{\prime} \simeq\langle y\rangle$. If $\mathbf{T}$ is the transversal, $\lim _{\mathbf{T}} A_{t} \simeq\langle x\rangle /\left\langle x^{t-1}\right\rangle \times\langle y\rangle$, and the morphism $\lim _{\longrightarrow} A_{t} \rightarrow G / G^{\prime}$ is clearly and isomorphism. Therefore $\mathcal{F}$ is independent.

Denote the active sum of the family $\mathcal{F}$ by $S$. To avoid confusion, denote by $a$ the generator in $S$ that corresponds to $x$, and $b_{i}$ the one that corresponds to $y x^{i(t-1)}$.

It is clear that we can define a homomorphism $f: G \rightarrow S$ such that $f(x)=a$ and $f(y)=b_{1}$. Clearly the diagram

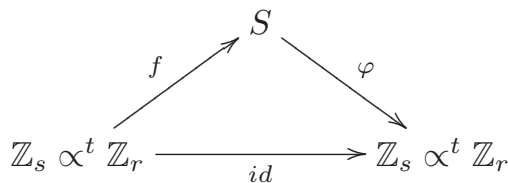

commutes. Thus, by Corollary $2.5, G$ is isomorphic to the active sum $S$.

\subsubsection{Coxeter Groups}

Let $W$ be a Coxeter group. We show that every $W$ is the active sum of subgroups of order 2. Following the notation of [22], we have a set $S$ of generators of $W$, where every $s \in S$ has order 2 . The relations are given by $\left(s s^{\prime}\right)^{m\left(s, s^{\prime}\right)}=1$. Define the family

$$
\mathcal{F}:=\left\{\left\langle s^{w}\right\rangle \mid w \in W, s \in S\right\} .
$$

Clearly, $\mathcal{F}$ is an active generating family of subgroups of $W$ of order 2 . We show first that $\mathcal{F}$ is regular and independent.

There is a homomorphism $W \rightarrow \mathbb{Z}_{2}$ sending every $s \in S$ to 1 . This means that for every $w \in W$ and $s \in S,\left\langle w^{-1} s w\right\rangle \cap W^{\prime}$ is trivial. Thus, $\mathcal{F}$ is regular.

Let $\Gamma$ be the graph obtained from the Coxeter graph of $(W, S)$ by deleting the edges labeled by $\infty$, or by an even number. Then $W / W^{\prime} \simeq \bigoplus_{\pi_{0}(\Gamma)} \mathbb{Z}_{2}$, where $\pi_{0}(\Gamma)$ denotes the connected components of $\Gamma$. Choose an $s_{c}$ in every element $c \in \pi_{0}(\Gamma)$. Then $T:=\left\{\left\langle s_{c}\right\rangle \mid c \in \pi_{0}(\Gamma)\right\}$ is a transversal for $\mathcal{F}$. It follows that $\lim _{c \in \pi_{0}(\Gamma)}\left\langle s_{c}\right\rangle \simeq$ $\bigoplus_{c \in \pi_{0}(\Gamma)} \mathbb{Z}_{2}$. Thus $\mathcal{F}$ is independent.

Let $H$ be the active sum of the family $\mathcal{F}$. To make the notation easier, assume that for every $F \in \mathcal{F}$ we have chosen an $s \in S$ and a $w \in W$ such that $F=\left\langle s^{w}\right\rangle$. Furthermore, if $F=\langle s\rangle$, assume that we have chosen $w=e$. Thus, we may suppose that the family $\mathcal{F}$ is of the form $\left\{\left\langle s_{j}^{w_{j}}\right\rangle\right\}_{j \in J}$ for some indexing set $J$. We have that $H$ can be presented by generators $\left\{x_{j}\right\}_{j \in J}$ and for every $j, k \in J$ a relation $x_{l}^{-1} x_{k}^{-1} x_{j} x_{k}$ if $s_{l}^{w_{l}}=s_{k}^{w_{k}} s_{j}^{w_{j}} s_{k}^{w_{k}}$ in $W$. Assume $S=\left\{s_{j}\right\}_{j \in J_{0}}$ with $J_{0} \subseteq J$, and that $x_{j}$ corresponds to $s_{j}$ for every $j \in J_{0}$. Define $\varphi: S \rightarrow H$ such that $\varphi\left(s_{j}\right)=x_{j}$. To be able to extend $\varphi$ to a homomorphism $W \rightarrow H$, we must show that the relations satisfied in $W$ by the elements of $S$ are also satisfied by their images under $\varphi$ in $H$. This is not hard, since the condition $\left(s s^{\prime}\right)^{m\left(s, s^{\prime}\right)}=1$ is equivalent to

$$
s^{\left(s^{\prime} s\right)^{\frac{m\left(s, s^{\prime}\right)-1}{2}}}=s^{\prime}
$$


if $m\left(s, s^{\prime}\right)$ is odd, and equivalent to

$$
s^{\prime\left(s s^{\prime}\right)^{\frac{m\left(s, s^{\prime}\right)-2}{2}} s}=s^{\prime}
$$

if $m\left(s, s^{\prime}\right)$ is even. Therefore we obtain a homomorphism $\varphi: W \rightarrow H$ that is clearly a section of the usual projection $H \rightarrow W$. By Corollary 2.5 we have that $H$ and $W$ are isomorphic.

\subsubsection{Best Wirtinger APproximations}

According to [39], a Wirtinger presentation is a presentation

$$
\left\langle\left\langle x_{i}\right\rangle_{i \in I} ;\left\langle r_{k}\right\rangle_{k \in K}\right\rangle
$$

where every relator $r_{k}$ is of the form $x_{i}^{-1} w^{-1} x_{j} w$ where $i, j \in I$ and $w$ is a word on the generators $\left\langle x_{i}\right\rangle_{i \in I}$. We will assume that there is a distinguished element $i_{0} \in I$. Given a group $G$ and an element $t \in G$, a Wirtinger approximation is a group $X$ given by a Wirtinger presentation $X=\left\langle\left\langle x_{i}\right\rangle_{i \in I} ;\left\langle r_{k}\right\rangle_{k \in K}\right\rangle$, and an epimorphism $\psi: X \rightarrow G$ such that $\psi\left(x_{i_{0}}\right)=t$, and $\psi$ induces an isomorphism $X / X^{\prime} \rightarrow G / G^{\prime}$. A best Wirtinger approximation is a Wirtinger approximation $\varphi: Y \rightarrow G$, with $Y=\left\langle\left\langle y_{p}\right\rangle_{p \in P} ;\left\langle r_{q}\right\rangle_{q \in Q}\right\rangle$, such that for every Wirtinger approximation $\psi: X \rightarrow G$, there exists an epimorphism $\widehat{\psi}: X \rightarrow Y$ such that $\widehat{\psi}\left(x_{i_{0}}\right)=y_{p_{0}}$ and $\psi=\varphi \circ \widehat{\psi}$.

Let $G$ be a group, and $t \in G$. Assume that $G / G^{\prime} \simeq \mathbb{Z}$ and that $G /\langle\langle t\rangle\rangle \simeq 1$, where $\langle\langle t\rangle\rangle$ denotes the normal closure of the subgroup generated by $t$. Theorem 1.3 in [39] shows that in such a case $(G, t)$ has a best Wirtinger approximation. We show that the active sum of the family $\mathcal{F}:=\left\{\left\langle t^{w}\right\rangle \mid w \in G\right\}$ together with the canonical projection is a best Wirtinger approximation. Since $G /\langle\langle t\rangle\rangle \simeq 1$, we have that $\mathcal{F}$ is a generating active family of subgroups of $G$. It is easily seen that $\mathcal{F}$ is regular and independent.

We may assume that $\mathcal{F}=\left\{\left\langle t^{w_{p}}\right\rangle\right\}_{p \in P}$ for some indexing set $P$, and furthermore, that $w_{p_{0}}=e$. Let $S$ be the active sum of the family $\mathcal{F}$, and $\varphi: S \rightarrow G$ the canonical projection. We can then present $S$ with generators $\left\{s_{p}\right\}_{p \in P}$, and for every $p, q \in P$ relations $s_{r}^{-1} s_{q}^{-1} s_{p} s_{q}$ whenever $\left(t^{w_{p}}\right)^{t^{w_{q}}}=t^{w_{r}}$ in $G$. Since $\mathcal{F}$ is regular and independent, we have that $\varphi: S \rightarrow G$ induces an isomorphism $S / S^{\prime} \rightarrow G / G^{\prime}$. We know that $\varphi$ is an epimorphism, and $\varphi\left(s_{p_{0}}\right)=t$. Thus $\varphi: S \rightarrow G$ is a Wirtinger approximation of $(G, t)$.

Assume that $\psi: X \rightarrow G$ is another Wirtinger approximation, with $X=\left\langle\left\langle x_{i}\right\rangle_{i \in I}\right.$; $\left.\left\langle r_{k}\right\rangle_{k \in K}\right\rangle$, as above. We want to construct an epimorphism $\gamma: X \rightarrow S$.

Since $X / X^{\prime} \simeq \mathbb{Z}$, we have that every $x_{i}$ is conjugate of $x_{i_{0}}$. Therefore $\psi\left(x_{i}\right)$ is a conjugate of $t$. There is a unique $p \in P$ such that $\psi\left(x_{i}\right)=t^{w_{p}}$. We can then define $\gamma\left(x_{i}\right)=s_{p}$ if $\psi\left(x_{i}\right)=t^{w_{p}}$. To be able to extend $\gamma$ to all of $X$, we must show that the relations in the presentation of $X$ are also satisfied by their images under $\gamma$. 
Assume that $x_{k}^{-1} x_{j}^{-1} x_{i} x_{j}$ is a relator in the presentation of $X$, and that $\psi\left(x_{i}\right)=t^{w_{p}}$, $\psi\left(x_{j}\right)=t^{w_{q}}$, and $\psi\left(x_{k}\right)=t^{w_{r}}$. Then we have

$$
1=\psi\left(x_{k}^{-1} x_{j}^{-1} x_{i} x_{j}\right)=\left(t^{w_{r}}\right)^{-1}\left(t^{w_{q}}\right)^{-1} t^{w_{p}} t^{w_{q}} .
$$

Therefore $s_{r}^{-1} s_{q}^{-1} s_{p} s_{q}$ is a relator in the presentation of $S$. We conclude that $\gamma: X \rightarrow$ $S$ is a homomorphism. Clearly, the diagram

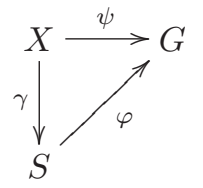

commutes, and $\gamma\left(x_{i_{0}}\right)=s_{p_{0}}$. It remains to show that $\gamma$ is an epimorphism. Since the morphisms $S / S^{\prime} \rightarrow G / G^{\prime}$ and $S / \operatorname{ker} \varphi \rightarrow G$, induced by $\varphi$ are isomorphisms, it is not hard to see that $X \rightarrow S \rightarrow S / S^{\prime}$ and $X \rightarrow S \rightarrow S / \operatorname{ker} \varphi \rightarrow S / Z(S)$ are epimorphisms, where the first arrow in both is $\gamma$ and the rest are projections. According to Corollary 2.2, we have that $\gamma$ is an epimorphism. Thus, $S$ is a best Wirtinger approximation of $(G, t)$.

\subsection{Atomic and molecular groups}

In this subsection we introduce a new class of subgroups, called atomic (or of weight 1 [24]). We also introduce the concept of molecular [35].

Definition 2.6. A group $G$ is called atomic if there exists an element $g \in G$, such that $G$ is equal to the normal closure of $\langle g\rangle$ in $G$. A subgroup $H$ of a group $G$ is called an atomic subgroup if $H$ is atomic in its own right. A group $G$ is called molecular if $G$ is the active sum of atomic subgroups.

We denote the normal closure of $\langle g\rangle$ in $G$ by $[g]_{G}$. Thus, $G$ is atomic if there is a $g \in G$ such that $[g]_{G}=G$.

Clearly, an abelian group is atomic if and only if it is cyclic. We also have that every simple group is atomic. It is not true in general that $G / G^{\prime}$ cyclic implies that $G$ is atomic [24]. However, it has been shown that for finite groups, $G$ is atomic if and only if $G / G^{\prime}$ is cyclic [26]. Thus symmetric groups are atomic. Every atomic group is of course molecular, as is any group that is the active sum of cyclic subgroups.

Since we know that for $n \geq 4$, the alternating group $A_{n}$ is not an active sum of cyclic subgroups, $A_{n}$ gives us an example of a molecular group that is not the active sum of cyclic subgroups.

\subsubsection{All atomic Class 2 Groups are CyCliC}

A class of groups in which being molecular is the same as being the active sum of cyclic subgroups is the class 2 groups. 
Lemma 2.7. If $G$ is a class 2 atomic group, then $G$ is cyclic.

Proof. Assume $G=[a]_{G}$. Then we have that $G / G^{\prime} \simeq\left\langle a G^{\prime}\right\rangle$. Since $G$ is class 2 , we have $G^{\prime} \subseteq Z(G)$. Therefore we can include $G / Z(G)$ in $G / G^{\prime}$, which is cyclic. We then use an exercise in [20] that says that $G / Z(G)$ cyclic implies $G$ abelian.

It is not hard to show that every subgroup of a class 2 group is a class 2 group. Thus we have:

Proposition 2.8. Let $G$ be a class 2 group. $G$ is molecular if and only if $G$ is the active sum of cyclic subgroups.

\subsubsection{All Finite ATOMiC P-GRoups ARE CYCLIC}

Another class for which being molecular is the same as being the active sum of cyclic subgroups is the class of $p$ groups.

Lemma 2.9. Let $p$ be a prime number. If $G$ is a finite atomic p-group, then $G$ is cyclic.

Proof. Since $G$ is atomic, $G / \Phi(G)$ is also atomic. Thus $G / \Phi(G)$ is cyclic. According to Corollary 1.2, page 173 [14] this means that $G$ is cyclic.

Thus we have:

Proposition 2.10. A p-group $G$ is molecular if and only if it is the active sum of a family of cyclic subgroups.

\subsection{The pullback of an active sum is an active sum}

We show that the pullback of a group that is the active sum of a family of subgroups is the active sum of the family of inverse images of the given family of subgroups.

\subsubsection{INVERSE IMAGE OF A FAMILY OF SUBGROUPS}

Let $\varphi: G \rightarrow H$ be an epimorphism of groups, and $\mathcal{H}=\left\langle H_{i}\right\rangle_{i \in I}$ a generating active family of subgroups of $H$. Assume that $0 \in I$, that $H_{0}$ is the trivial subgroup, and that $H_{0} \leq H_{i}$ for all $i \in I$. We can produce a generating active family of subgroups of $G$ by considering the inverse images of the subgroups $H_{i}$. Specifically, let $F_{i}=\varphi^{-1} H_{i}$ and consider the family $\mathcal{F}=\left\langle F_{i}\right\rangle_{i \in I}$. The order is given by $F_{i} \leq F_{j}$ if and only if $H_{i} \leq H_{j}$ in $\mathcal{H}$. Clearly, $F_{0}=\operatorname{ker}(\varphi)$. Let $S$ be the active sum of the family $\mathcal{F}, S^{\prime}$ the active sum of the family $\mathcal{H}$ and $\xi: S^{\prime} \rightarrow H$ the canonical epimorphism. We will show that the pullback of $\xi$ along $\varphi$ is isomorphic to the active sum of the family $\mathcal{F}$. We will need the following lemma. 
Lemma 2.11. Suppose that $h_{1} \cdot h_{2} \cdots h_{n}=k_{1} \cdot k_{2} \cdots k_{m} \in * \mathcal{H}$, with $h_{i} \in H_{r_{i}}$ and $k_{j} \in H_{s_{j}}$. If for every $i=1, \ldots, n$, we have $g_{i} \in F_{r_{i}}$ such that $\varphi\left(g_{i}\right)=h_{i}$, then we can find, for every $j=1, \ldots$, m elements $g_{j}^{\prime} \in F_{s_{j}}$ such that $\varphi\left(g_{j}^{\prime}\right)=k_{j}$ and $g_{1} \cdot g_{2} \cdots g_{n}=g_{1}^{\prime} \cdot g_{2}^{\prime} \cdots g_{n}^{\prime}$ in the active sum of $\mathcal{F}$.

Proof. If $h_{1} \cdot h_{2} \cdots h_{n}=k_{1} \cdot k_{2} \cdots k_{m} \in * \mathcal{H}$, then there is a sequence $h_{1} \cdot h_{2} \cdots h_{n}=$ $w_{0} \sim w_{1} \sim \cdots \sim w_{t}=k_{1} \cdot k_{2} \cdots k_{m}$ of words in the elements of the family $\mathcal{H}$, where the symbol $w \sim w^{\prime}$ means that $w$ can be obtained from $w^{\prime}$ by adding the identity of some $H_{i}$ between two letters of $w$, or by multiplying together two consecutive letters of $w$ that belong to the same group, or by the opposite operations (deleting an identity that appears in $w$ or considering a product in some $H_{i}$ as two letters). It suffices then, to show that the claim of the lemma is true if $h_{1} \cdot h_{2} \cdots h_{n} \sim k_{1} \cdot k_{2} \cdots k_{m}$. We do the case where $h_{\ell}=x y$ in $H_{i}$ and leave all the other cases to the reader. We have $h_{1} \cdot h_{2} \cdot h_{\ell-1} \cdot(x y) \cdot h_{\ell+1} \cdots h_{n} \sim h_{1} \cdot h_{2} \cdot h_{\ell-1} \cdot x \cdot y \cdot h_{\ell+1} \cdots h_{n}$. Assume $\varphi\left(g_{i}\right)=h_{i}$. Then $\varphi\left(g_{\ell}\right)=x y$. Since $\varphi$ is onto, we can find $\hat{x}, \hat{y} \in F_{r_{\ell}}$ such that $\varphi(\hat{x})=x$ and $\varphi(\hat{y})=y$. Notice that $g_{\ell} \hat{y}^{-1} \hat{x}^{-1} \in \operatorname{ker} \varphi \subseteq F_{r_{\ell}}$. Therefore $g_{\ell} \hat{y}^{-1}=g_{\ell} \hat{y}^{-1} \hat{x}^{-1} \hat{x} \in F_{r_{\ell}}$. We have $\varphi\left(g_{\ell} \hat{y}^{-1}\right)=x, \varphi(\hat{y})=y$ and $\left(g_{\ell} \hat{y}^{-1}\right) \hat{y}=g_{\ell}$. Therefore, the elements we can choose are $g_{1}, \ldots, g_{\ell-1}, g_{\ell} \hat{y}^{-1}, \hat{y}, g_{\ell+1}, \ldots g_{n}$.

\subsubsection{The PUllbaCK OF AN ACTIVE SUM}

Theorem 2.12. With the same definitions, consider the pullback

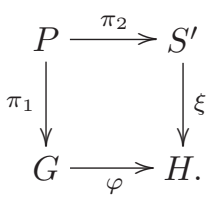

Then $P$ is isomorphic to the active sum of the family $\mathcal{F}$.

Proof. Let $\zeta: S \rightarrow G$ be the canonical epimorphism. Observe that the family $\left\langle\left.\varphi\right|_{F_{i}}\right.$ : $\left.F_{i} \rightarrow H_{i}\right\rangle$ induces a homomorphism $\psi: S \rightarrow S^{\prime}$ such that the diagram

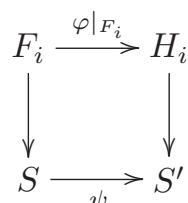

commutes, where the vertical arrows are the canonical injections. It is easy to see 
that the outer square of the diagram

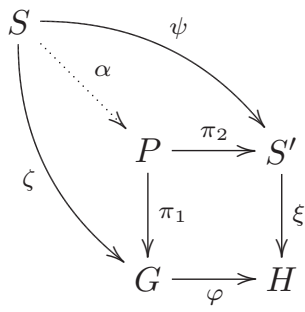

commutes. Therefore, we can induce a unique homomorphism $\alpha: S \rightarrow P$ such that the triangles in the above diagram commute.

Now the opposite direction. Let $R$ and $R^{\prime}$ the appropriate normal subgroups of $\star \mathcal{F}$ and $* \mathcal{H}$ respectively, such that $S=\star \mathcal{F} / R$ and $S^{\prime}=\star \mathcal{F} / R^{\prime}$.

We claim that, for every $\left(g, h_{1} h_{2} \cdots h_{n} R^{\prime}\right) \in P$, with $h_{r} \in H_{i_{r}}$, we can find $g_{r} \in F_{i_{r}}$ such that $\varphi\left(g_{r}\right)=h_{r}$ and $g=g_{1} g_{2} \cdots g_{n}$.

Since $\varphi$ is an epimorphism, we can find $g_{r}^{\prime} \in F_{i_{r}}$ such that $\varphi\left(g_{r}^{\prime}\right)=h_{r}$. Since $\left(g, h_{1} h_{2} \cdots h_{n} R^{\prime}\right) \in P$, we have that

$$
\varphi(g)=\xi\left(h_{1} h_{2} \cdots h_{n} R^{\prime}\right)=h_{1} h_{2} \cdots h_{n}=\varphi\left(g_{1}^{\prime} g_{2}^{\prime} \cdots g_{n}^{\prime}\right) .
$$

This means that $g g_{n}^{\prime-1} g_{n-1}^{\prime}{ }^{-1} \cdots g_{1}^{\prime-1} \in F_{0}=k e r \varphi$. Therefore

$$
g g_{n}^{\prime-1} g_{n-1}^{-1} \cdots g_{1}^{\prime-1} g_{1}^{\prime} \in F_{i_{1}}
$$

and clearly

$$
\varphi\left(g g_{n}^{\prime-1} g_{n-1}^{-1} \cdots g_{1}^{\prime-1} g_{1}^{\prime}\right)=\varphi\left(g_{1}^{\prime}\right)=h_{1} .
$$

So, defining $g_{1}=g g_{n}^{\prime-1} g_{n-1}^{-1} \cdots g_{1}^{\prime-1} g_{1}^{\prime}$ and $g_{r}=g_{r}^{\prime}$ if $r>1$, we have $g=g_{1} g_{2} \cdots g_{n}$ and $\varphi\left(g_{i}\right)=h_{i}$ for all $i$.

Define $\beta: P \rightarrow S$ as follows: given $\left(g, h_{1} h_{2} \cdots h_{n} R^{\prime}\right) \in P$ with $h_{r} \in H_{i_{r}}$, choose $g_{r} \in F_{i_{r}}$ such that $\varphi\left(g_{r}\right)=h_{r}$ for every $r=1, \ldots, n$ and such that $g=g_{1} \cdots g_{n}$. Put $\beta\left(g, h_{1} h_{2} \cdots h_{n} R^{\prime}\right)=g_{1} g_{2} \cdots g_{n} R$. We must show that this definition does not depend on the choice of the $g_{i}$ or on the choice of $h_{1} \cdots h_{n} R^{\prime}$.

Assume first that $g_{r}^{\prime} \in F_{i_{r}}$ are such that $\varphi\left(g_{r}^{\prime}\right)=h_{r}$, and $g=g_{1}^{\prime} g_{2}^{\prime} \cdots g_{n}^{\prime}$. We then have that $g_{r} g_{r}^{\prime-1} \in \operatorname{ker} \varphi$ for ever $r$. Let $x_{i} \in \operatorname{ker} \varphi$ such that $g_{i}^{\prime}=x_{i} g_{i}$. Taking into 
account that $\operatorname{ker} \varphi=F_{0} \leq F_{i}$ for all $i \in I$, we have

$$
\begin{aligned}
& g_{1}^{\prime} g_{2}^{\prime} \cdots g_{n}^{\prime} R=\left(x_{1} g_{1}\right) \cdots\left(x_{n-1} g_{n-1}\right)\left(x_{n} g_{n}\right) R \\
& =\left(x_{1} g_{1}\right) \cdots\left(x_{n-1} g_{n-1} x_{n}\right) g_{n} R \\
& =\left(x_{1} g_{1}\right) \cdots\left(x_{n-1} x_{n}^{g_{n-1}^{-1}} g_{n-1}\right) g_{n} R \\
& =\left(x_{1} g_{1}\right) \cdots\left(x_{n-2} g_{n-2} x_{n-1} x_{n}^{g_{n-1}^{-1}}\right) g_{n-1} g_{n} R \\
& =\left(x_{1} g_{1}\right) \cdots\left(x_{n-2} x_{n-1}^{g_{n-2}^{-1}} x_{n}^{g_{n-1}^{-1} g_{n-2}^{-1}} g_{n-2}\right) g_{n-1} g_{n} R \\
& =\left(x_{1} x_{2}^{g_{1}^{-1}} x_{3}^{g_{2}^{-1} g_{1}^{-1}} \cdots x_{n}^{g_{n-1}^{-1} \cdots g_{2}^{-1} g_{1}^{-1}}\right) g_{1} g_{2} \cdots g_{n} R \text {. }
\end{aligned}
$$

Applying $\zeta: S \rightarrow G$ to both sides we obtain

$$
\begin{aligned}
g=g_{1}^{\prime} g_{2}^{\prime} \cdots g_{n}^{\prime} & =\left(x_{1} x_{2}^{g_{1}^{-1}} x_{3}^{g_{2}^{-1} g_{1}^{-1}} \cdots x_{n}^{g_{n-1}^{-1} \cdots g_{2}^{-1} g_{1}^{-1}}\right) g_{1} g_{2} \cdots g_{n} \\
& =\left(x_{1} x_{2}^{g_{1}^{-1}} x_{3}^{g_{2}^{-1} g_{1}^{-1}} \cdots x_{n}^{g_{n-1}^{-1} \cdots g_{2}^{-1} g_{1}^{-1}}\right) g
\end{aligned}
$$

in $G$. Therefore $x_{1} x_{2}^{g_{1}^{-1}} x_{3}^{g_{2}^{-1} g_{1}^{-1}} \cdots x_{n}^{g_{n-1}^{-1} \cdots g_{2}^{-1} g_{1}^{-1}}$ is the identity. We conclude that $g_{1}^{\prime} g_{2}^{\prime} \cdots g_{n}^{\prime} R=g_{1} g_{2} \cdots g_{n} R$.

We show now that the definition of $\beta$ does not depend on the choice of

$$
h_{1} h_{2} \cdots h_{n} R^{\prime} .
$$

Assume that $\left(g, h_{1} h_{2} \cdots h_{n} R^{\prime}\right)=\left(g, h_{1}^{\prime} h_{2}^{\prime} \cdots h_{n}^{\prime} R^{\prime}\right)$. Therefore we have that

$$
h_{1} h_{2} \cdots h_{n} h_{m}^{\prime-1} \cdots h_{1}^{\prime-1} \in R^{\prime}
$$

This means that we can write $h_{1} \cdot h_{2} \cdots h_{n} \cdot h_{m}^{\prime}{ }^{-1} \cdots h_{1}^{\prime-1}$ in $\star \mathcal{H}$ as a product of conjugates of generators of $R^{\prime}$. It is clear that for every factor in this last product we can find a preimage along $\varphi$ in such a way that the resulting product in $\nsucc \mathcal{F}$ is an element of $R$. Using Lemma 2.11 we can find elements $g_{i} \in F_{r_{i}}$ and $g_{j}^{\prime} \in F_{s_{j}}$ for all $i, j$, such that $\varphi\left(g_{i}\right)=h_{i}, \varphi\left(g_{j}^{\prime}\right)=h_{j}$ and $g_{1} g_{2} \cdots g_{n} g_{m}^{\prime-1} \cdots g_{1}^{\prime-1} R=R$. That is to say $g_{1} g_{2} \cdots g_{n} R=g_{1}^{\prime} g_{2}^{\prime} \cdots g_{m}^{\prime} R$.

\subsubsection{Finite Groups AS ACTIVE SUMS OF PROPER SUbGroups}

We draw now some conclusions of the pullback theorem above. For any group $G$, we can decompose $G / G^{\prime}$ as a direct sum of cyclic groups $G / G^{\prime} \simeq \bigoplus_{i \in I} C_{i}$. Let $\varphi: G \rightarrow \bigoplus_{i \in I} C_{i}$ be the projection. We may assume that $0 \in I$ and $C_{0}$ is trivial. Notice then that $G / G^{\prime}$ is the active sum of the family $\left\langle C_{i}\right\rangle_{i \in I}$, where $C_{0} \leq C_{i}$ for all $i \in I$. Theorem 2.12 asserts that $G$ is isomorphic to the active sum of the family 
$\left\langle\varphi^{-1}\left(C_{i}\right)\right\rangle_{i \in I}$. This produces a non-trivial decomposition of $G$ as an active sum of normal subgroups provided that $G / G^{\prime}$ is not cyclic.

As pointed out in Section 2.3, a finite group $G$ is atomic if and only if $G / G^{\prime}$ is cyclic. Thus we have:

Theorem 2.13. Every non-atomic finite group is the active sum of proper normal subgroups.

The following theorem states that, except for cyclic groups of prime power order, every finite group is decomposable.

Theorem 2.14. If $G$ is a finite group that is not a cyclic p-group, then $G$ is the active sum of proper subgroups.

Proof. The previous theorem allows us to assume that $G$ is atomic. By Lemma 2.9, $G$ cannot be a $p$-group. Then $G$ is the active sum of its subgroups of order a prime [35].

\subsection{Central extensions and homology}

\subsubsection{Homology, GaneA's maP AND REGUlarity AND INDEPENDENCE}

Recall that a central extension is a short exact sequence

$$
K>\stackrel{\kappa}{\rightarrow} S \stackrel{\pi}{\rightarrow} G
$$

where $K \subset Z(G)$. Such an extension gives rise to the five-term exact sequence [21], [4]:

$$
H_{2}(S) \stackrel{\pi^{*}}{\longrightarrow} H_{2}(G) \longrightarrow K \longrightarrow H_{1}(S) \stackrel{\pi^{*}}{\longrightarrow} H_{1}(G) \longrightarrow 0 \text {. }
$$

Therefore, $K$ is trivial, that is, $S \rightarrow G$ is an isomorphism, if and only if $H_{1}(S) \rightarrow$ $H_{1}(G)$ is monic and $H_{2}(S) \rightarrow H_{2}(G)$ is epic.

Thus, if $S$ is the active sum of a generating active family $\mathcal{F}$ of subgroups of $G$, the canonical map $S \rightarrow G$ is an isomorphism if and only if $\mathcal{F}$ is regular and independent, and $H_{2}(S) \rightarrow H_{2}(G)$ is surjective. As a consequence we have:

Lemma 2.15. If $G$ is a group such that $H_{2}(G)$ is trivial, then every generating active family of subgroups of $G$ that is regular and independent has active sum $G$.

Still assuming $S$ to be the active sum of a family $\mathcal{F}$ of subgroups of $G$, the image of the homomorphism $H_{2}(S) \rightarrow H_{2}(G)$ contains, besides the images of the inclusion induced homomorphisms $H_{2}(F) \rightarrow H_{2}(G)$ with $F \in \mathcal{F}$, the image of the so called Ganea map $\chi: H_{1}(G) \otimes Z(G) \rightarrow H_{2}(G)$ which we describe next.

If $g, g^{\prime} \in G$ are such that $g g^{\prime}=g^{\prime} g$, an element $g \wedge g^{\prime} \in H_{2}(G)$, the Pontrjagin product, is defined as follows. Define the homomorphism $\mathbb{Z} \oplus \mathbb{Z} \rightarrow G$ such that $(1,0) \mapsto g$ and $(0,1) \mapsto g^{\prime}$. We thus obtain $\mathbb{Z} \simeq H_{2}(\mathbb{Z} \oplus \mathbb{Z}) \rightarrow H_{2}(G)$. One generator 
of $H_{2}(\mathbb{Z} \oplus \mathbb{Z})$ goes to the element $g \wedge g^{\prime}$ and the other to its inverse. This ambiguity of sign can be avoided using the Schur-Hopf formula [4]. However, the above description of $g \wedge g^{\prime}$ will be enough for our purposes.

Assume now that we have a central extension

$$
N>\stackrel{\kappa}{\rightarrow} G \stackrel{\pi}{\rightarrow} Q
$$

Ganea [12] has added a term to the exact sequence of five terms:

$$
H_{1}(G) \otimes N \stackrel{\chi}{\rightarrow} H_{2}(G) \stackrel{\pi_{*}}{\longrightarrow} H_{2}(Q) \rightarrow N \rightarrow H_{1}(G) \stackrel{\pi_{*}}{\rightarrow} H_{1}(Q) \rightarrow 0 .
$$

We will call $\chi$ Ganea's map. Using the algebraic description of Ganea's map given in [9], it follows that $\chi([g] \otimes x)=g \wedge x$. Clearly $\chi$ is an epimorphism if and only if $H_{2}(G)$ is generated by elements of the form $g \wedge x$ with $g \in G$ and $x \in N$. We can take $N=Z(G)$ in particular.

Theorem 2.16. If $\chi: H_{1}(G) \otimes Z(G) \rightarrow H_{2}(G)$ is an epimorphism, then every generating active family of subgroups of $G$ that is regular and independent has active sum isomorphic to $G$.

Proof. Let $\mathcal{F}$ be a regular and independent generating active family of subgroups of $G$, and let $S$ be the active sum of the family $\mathcal{F}$. Let $\varphi: S \rightarrow G$ be the induced epimorphism and let $M$ be the kernel of $\varphi$. Consider the short exact sequence

$$
0 \rightarrow M \rightarrow S \stackrel{\varphi}{\rightarrow} G \rightarrow 0
$$

We obtain the five term exact sequence

$$
H_{2}(S) \stackrel{\varphi_{*}}{\rightarrow} H_{2}(G) \rightarrow M \rightarrow H_{1}(S) \stackrel{\varphi_{*}}{\rightarrow} H_{1}(G) \rightarrow 0 .
$$

Since $\mathcal{F}$ is regular and independent, we have that $\varphi_{*}$ is an isomorphism at the $H_{1}$ level. To show that $M$ is the trivial group it suffices then to show that $\varphi_{*}$ is an epimorphism at the $H_{2}$ level. Since $H_{2}(G)$ is generated by elements of the form $g \wedge x$ with $g \in G$ and $x \in Z(G)$, it will be enough if we show that these elements are in the image of $\varphi_{*}$. Let $\widehat{g}, \widehat{x} \in S$ such that $\varphi(\widehat{g})=g$ and $\varphi(\widehat{x})=x$. Since $x \in Z(G)$, Lemma 1.1.5 tells us that $\widehat{x} \in Z(S)$. Therefore $\widehat{g} \wedge \widehat{x} \in H_{2}(S)$. We have that $\varphi_{*}(\widehat{g} \wedge \widehat{x})=g \wedge x$. We conclude that $M$ is the zero group, that is, $\varphi: S \rightarrow G$ is an isomorphism.

Recall that the short exact sequence (1) is called stem if it is central and $N \subset G^{\prime}$. As a corollary to our last result we have:

Corollary 2.17. Let $N \gg \stackrel{\kappa}{\rightarrow} G \stackrel{\pi}{\rightarrow} Q$ be a stem extension, with $H_{2}(Q)$ finitely generated and $\mathrm{N}$ isomorphic to $\mathrm{H}_{2}(Q)$. If $\mathcal{F}$ is a generating active family of subgroups of $G$ that is regular and independent, then the active sum of the family $\mathcal{F}$ is isomorphic to $G$. 
Proof. Since we have a central extension, we can induce the long exact sequence (2). Since the extension is stem, we have that $\pi_{*}: H_{1}(G) \rightarrow H_{1}(Q)$ is an isomorphism [9]. This means that the next map to the left is trivial. Therefore, the homomorphism $H_{2}(Q) \rightarrow N$ is an epimorphism. Since $H_{2}(Q)$ is finitely generated and isomorphic to $N$, this map must be an isomorphism. This means that $\pi_{*}$ at the level $H_{2}$ is trivial. It follows that Ganea's map $\chi$ is an epimorphism. Therefore, the map $H_{1}(G) \otimes Z(G) \rightarrow$ $H_{2}(G)$ is surjective and Theorem 2.16 finishes the proof.

\subsubsection{Example: Groups of ORder $p^{3}$}

As an application we will see that all groups of order $p^{3}$, with $p \neq 2$ prime, are active sums of cyclic groups. According to Theorem 5.1, p. 203 of [14], there are only two such groups for every $p \neq 2$, namely:

$$
H=\left\langle x, y, z \mid x^{p}=y^{p}=z^{p}=1,[x, z]=[y, z]=1,[x, y]=z\right\rangle,
$$

and

$$
K=\left\langle a, b \mid a^{p^{2}}=b^{p}=1,[a, b]=a^{p}\right\rangle,
$$

and they are extra special. Actually $H^{\prime}=Z(H)=\langle z\rangle$, and $K^{\prime}=Z(K)=\left\langle a^{p}\right\rangle$. Since $H / H^{\prime} \simeq K / K^{\prime} \simeq \mathbb{Z}_{p} \oplus \mathbb{Z}_{p}$ and $H_{2}\left(\mathbb{Z}_{p} \oplus \mathbb{Z}_{p}\right) \simeq \mathbb{Z}_{p}$, we see that both groups can be fitted in Corollary 2.17. Therefore, to show that they can be expressed as an active sum of cyclic subgroups, all we have to do is to find an active generating family $\mathcal{F}$ of cyclic subgroups that is regular and independent. In the case of $H$ we consider the family

$$
\mathcal{F}=\left\{\left\langle x z^{i}\right\rangle \mid i=0,1, \ldots, p-1\right\} \cup\left\{\left\langle y z^{i}\right\rangle \mid i=0,1, \ldots, p-1\right\},
$$

and in the case of $K$ we consider

$$
\mathcal{G}=\{\langle a\rangle\} \cup\left\{\left\langle b a^{i p}\right\rangle \mid i=0,1, \ldots, p-1\right\}
$$

We leave it to the reader to show that $\mathcal{F}$ and $\mathcal{G}$ are regular and independent.

\section{Perfect and simple groups}

\subsection{Regularity and independence for perfect groups}

We will show that most of the groups $\mathrm{SL}_{2}(q)$, all the simple groups with trivial Schur multiplier and all of Suzuki's groups [41], with the exception of the smallest one, are active sums of cyclic subgroups. The proofs consist on identifying regular and independent generating active families of cyclic subgroups (actually, one such subgroup and all its conjugates), and considering the Schur multiplier, that turns out to be trivial in many of these cases.

If $G$ is a perfect group, independence is not an issue: 
Lemma 3.1. If $\mathcal{F}$ is a generating active family of subgroups of a perfect group $G$, then $\mathcal{F}$ is independent.

Proof. If $G$ is a perfect group, then $G / G^{\prime}$ is trivial. On the other hand, if we take any $F \in \mathcal{F}$, we have that $F G^{\prime} / G^{\prime}$ is also trivial. Thus the corresponding colimit in the definition of independence is the trivial group. Since $G / G^{\prime}$ is also trivial, the family is independent.

Next, we have a condition for regularity for cyclic subgroups:

Proposition 3.2. Let $G$ be a group. If $a, b \in G, a \neq 0$, are such that $a^{b}=a^{s}$, and $s-1$ and the order of a are relative prime, then the family

$$
\mathcal{F}=\left\{w^{-1}\langle a\rangle w \mid w \in G\right\}
$$

is a regular (not necessarily generating) active family of subgroups of $G$.

Proof. $\mathcal{F}$ is clearly an active family of subgroups of $G$. Observe that

$$
a^{s-1}=a^{-1} b^{-1} a b \in\left[\langle a\rangle, N_{G}(\langle a\rangle)\right] .
$$

Since $(o(a), s-1)=1$, then $\langle a\rangle=\left\langle a^{s-1}\right\rangle$. Therefore $\langle a\rangle=\left[\langle a\rangle, N_{G}(\langle a\rangle)\right]$. Since every $F \in \mathcal{F}$ is a conjugate of $\langle a\rangle$, we have that $F=\left[F, N_{G}(F)\right]$ is true for every $F \in \mathcal{F}$. According to Lemma $1.1 .9, \mathcal{F}$ is regular.

Remark 3.3. Under the hypotheses of the proposition, if $\mathcal{F}$ is generating, then $G$ is perfect (every element of the family is contained in $G^{\prime}$ ). If $G$ is simple, then the family $\mathcal{F}$ is a generating family.

It is clear that the conditions for the proposition are satisfied if a simple group $G$ has as a subgroup a dihedral group of order $2 m$, with $m$ odd. In such a case $G$ has a regular generating active family of cyclic subgroups of order $m$.

\subsection{Simple groups with trivial Schur multiplier}

Theorem 3.4. Every finite simple group $G$ with trivial Schur multiplier is the active sum of cyclic subgroups.

Proof. Assume $G$ is a finite non-abelian simple group. Then any non-trivial active family of cyclic subgroups is generating, and by Lemma 3.1, independent. If in addition $H_{2}(G)$ is trivial, Lemma 2.2.15 tells us that $G$ is the active sum of any non-trivial regular active family of cyclic subgroups of $G$. It suffices then to find elements in $G$ that satisfy the conditions of Proposition 3.2. Observe that it suffices to show that $G$ contains a dihedral subgroup of order $2 n$ with $n$ odd. Since $G$ is simple it is generated by a conjugacy class of involutions. According to Corollary 2.67 in [15] there must be involutions $s, t \in G$ such that the order of $s t$ is not a power of 2. Assume the order of $s t$ is $2^{k} n$ with $n>1$ odd. Then $\left\langle s,(s t)^{2^{k}}\right\rangle$ is isomorphic to a dihedral subgroup of order $2 n$ 
The above proof tells us nothing about the size of the cyclic subgroups that have to be considered.

\subsection{Special linear groups}

3.3.1. $\mathrm{SL}_{n}(q), n \geq 3$

Theorem 3.5. Let $n \geq 3$. The group $\mathrm{SL}_{n}(q)$ is an active sum of subgroups of order 3, if it is not one of the following: $\mathrm{SL}_{3}(2), \mathrm{SL}_{3}(3), \mathrm{SL}_{4}(2)$ and $\mathrm{SL}_{3}(4)$.

Proof. According to [23], pages 244-246, the groups $\mathrm{SL}_{n}(q)$ are perfect and have trivial Schur multiplier (we are assuming that $n \geq 3$ and that the group is not one of $\mathrm{SL}_{3}(2), \mathrm{SL}_{3}(3), \mathrm{SL}_{4}(2)$ or $\left.\mathrm{SL}_{3}(4)\right)$. [11] in page 2 gives for any field $K$ two matrices of order 2 in $\mathrm{SL}_{3}(K)$ that generate an $S_{3}$ :

$$
A:=\left(\begin{array}{ccc}
-1 & 0 & 0 \\
0 & 0 & 1 \\
0 & 1 & 0
\end{array}\right), \quad B:=\left(\begin{array}{ccc}
0 & 1 & 0 \\
1 & 0 & 0 \\
0 & 0 & -1
\end{array}\right) .
$$

Furthermore, $\mathrm{SL}_{k-1}(q)$ is included in $\mathrm{SL}_{k}(q)$. Thus we conclude that $S_{3}$ is a subgroup of $\mathrm{SL}_{n}(q)$. Using Lemma 3.1 and Proposition 3.2, we obtain a generating regular and independent active family of subgroups of order 3. Since the Schur multiplier is trivial, the active sum of the family is isomorphic to $\mathrm{SL}_{n}(q)$, according to Lemma 2.2.15.

We will see in the sequel to this paper, that none of $\mathrm{SL}_{3}(2), \mathrm{SL}_{3}(3), \mathrm{SL}_{4}(2)$ and $\mathrm{SL}_{3}(4)$ is an active sum of cyclic subgroups.

\subsection{2. $\mathrm{SL}_{2}(q), q$ A POWER OF A PRIME}

Let $p$ be a prime number, and let $q=p^{r}$ be a power of $p, r \geq 1$. We denote by $\mathbb{F}_{q}$ the finite field of order $q$. We will consider the special linear groups $\mathrm{SL}_{2}(q)$ of $2 \times 2$ matrices with entries in $\mathbb{F}_{q}$ and determinant 1 . The theorem we want to prove is:

Theorem 3.6. If $q \neq 4$ then the group $\mathrm{SL}_{2}(q)$ is an active sum of cyclic subgroups.

Observe that $\mathrm{SL}_{2}(4) \simeq A_{5}$. We show in the sequel to this paper, that $A_{5}$ is not the active sum of cyclic subgroups. We begin with the case where $q-1$ is not a power of 2 .

Proposition 3.7. If $q-1$ is not a power of 2 , and $q \neq 4$, then the group $\mathrm{SL}_{2}(q)$ is an active sum of cyclic subgroups.

Proof. According to [23], pg. 244 and 246, $\mathrm{SL}_{2}(q)$ is a perfect group, except for $\mathrm{SL}_{2}(2)$, and the Schur multiplier is trivial (with the exception of $\mathrm{SL}_{2}(4)$ ). $\mathrm{SL}_{2}(2) \simeq S_{3}$, and this last group is the active sum of cyclic subgroups. Assume that $q \neq 2$. It follows from Lemma 2.2.15, that all we have to do is to find a regular and independent 
generating active family of cyclic subgroups. According to Lemma 3.1, independence is not an issue. We will find elements in $\mathrm{SL}_{2}(q)$ satisfying the conditions of Proposition 3.2. Let $t$ be a prime divisor of $q-1$ with $t \neq 2$, and let $\lambda$ be a primitive root of $F_{q}^{*}$. Consider the following elements of $\mathrm{SL}_{2}(q)$ :

$$
A:=\left(\begin{array}{cc}
\lambda^{\frac{q-1}{t}} & 0 \\
0 & \lambda^{-\frac{q-1}{t}}
\end{array}\right), \quad B:=\left(\begin{array}{cc}
0 & -1 \\
1 & 0
\end{array}\right) .
$$

Observe that the order of $A$ is $t$, and that $A^{B}=A^{-1}$. Since $t$ is a prime number different from 2, $A$ and $B$ satisfy the conditions of Proposition 3.2. Since $A$ is not in the center of $\mathrm{SL}_{2}(q)$, it follows from 3.2.8 in [37] that the family consisting of the cyclic subgroup $\langle A\rangle$ and its conjugates is a generating family. Thus, $\mathrm{SL}_{2}(q)$ is an active sum of cyclic subgroups of order $t$.

Proposition 3.8. If $p \neq 3$ and $q-1$ is a power of 2 , then $\mathrm{SL}_{2}(q)$ is an active sum of cyclic subgroups.

Proof. Again [23] tells us that $\mathrm{SL}_{2}(q)$ is perfect, and its Schur multiplier is trivial. Consider the elements

$$
A:=\left(\begin{array}{ll}
1 & 0 \\
1 & 1
\end{array}\right), \quad B:=\left(\begin{array}{cc}
c c 2 & 0 \\
0 & 2^{-1}
\end{array}\right) .
$$

Since $p \neq 2, B$ is well defined. We have that $A^{B}=A^{4}$. Since $o(A)=p$ and 3 are relative prime, we have that $A$ and $B$ satisfy the hypotheses of Proposition 3.2. Therefore the family consisting of the subgroups that are conjugates of the one generated by $A$, is a regular and independent family of cyclic subgroups. Since $A$ is not in the center of $\mathrm{SL}_{2}(q)$, that the family is generating follows as in the previous proposition.

The above propositions, together with the observation about $\mathrm{SL}_{2}(4)$, leave us with the cases $\mathrm{SL}_{2}\left(3^{r}\right)$, where $3^{r}-1$ is a power of 2 . According to [19] it was Leo Hebreus who showed that the equation $2^{x}-3^{y}=-1$ has only two solutions, namely $2^{1}-3^{1}$ and $2^{3}-3^{2}$. Thus we are left with $\mathrm{SL}_{2}(3)$ and $\mathrm{SL}_{2}(9)$. We will show that both groups are active sum of cyclic subgroups.

Proposition 3.9. The group $\mathrm{SL}_{2}(3)$ is the active sum of subgroups of order 3 .

Proof. The order of $G:=S L_{2}(3)$ is $24 . G^{\prime}$ has order 8. It follows that for any element $A$ of order $3,\langle A\rangle \cap G^{\prime}=1$. It follows from Lemma 1.1.9, that the family $\mathcal{F}$ consisting of all the subgroups of $G$ of order 3 , is regular. Since $\mathcal{F}$ consists of 3 -Sylow subgroups of $G$, then a transversal for $\mathcal{F}$ has exactly one element, $\langle A\rangle$ say. Clearly, the order of $\langle A\rangle G^{\prime}$ is 24 , therefore $\langle A\rangle G^{\prime} / G^{\prime} \simeq G / G^{\prime}$, and the family is independent. [23] again says that $G$ has trivial Schur multiplier. By Lemma $2.2 .15, G$ is the active sum of $\mathcal{F}$ if we can show that the family is generating. $\mathrm{SL}_{2}(3)$ has four 3 -Sylow subgroups, giving 8 elements of order 3 . It is not hard to produce with these ones five more elements to show that the family does generate. 
Proposition 3.10. The group $\mathrm{SL}_{2}(9)$ is the active sum of subgroups of order 3 .

Proof. Consider the family $\mathcal{F}$ consisting of the cyclic group generated by

$$
B:=\left(\begin{array}{ll}
1 & 1 \\
0 & 1
\end{array}\right)
$$

and all its conjugates. Every element of $\mathcal{F}$ has order 3 . Since $\mathrm{SL}_{2}(9)$ is perfect, we have that the family is independent. Let $i$ be a square root of -1 in $\mathbb{F}_{9}$, and consider the following element in $\mathrm{SL}_{2}(9)$ :

$$
X:=\left(\begin{array}{cc}
-i & 0 \\
0 & i
\end{array}\right) .
$$

Since $B^{X}=B^{-1}$, then $\mathcal{F}$ is regular. And by 3.2.8 in [37], the family generates.

Let $S$ be the active sum of the family $\mathcal{F}$, and let $\varphi: S \rightarrow \mathrm{SL}_{2}(9)$ be the projection. To prove that $\varphi$ is an isomorphism, we will show that $\varphi_{*}: H_{2}(S) \rightarrow H_{2}\left(\mathrm{SL}_{2}(9)\right)$ is an epimorphism. According to [23], pg. $246, H_{2}\left(\mathrm{SL}_{2}(9)\right) \simeq \mathbb{Z}_{3}$. Let $\lambda \in \mathbb{F}_{q}$ such that $\lambda \notin\{0,1,2\}$. Define

$$
A:=\left(\begin{array}{cc}
1 & \lambda \\
0 & 1
\end{array}\right)
$$

Observe that $A$ is an element of $\mathrm{SL}_{2}(9)$ of order 3 , and that $A B=B A$. Thus $P:=\langle B, A\rangle$ is a 3 -Sylow subgroup of $\mathrm{SL}_{2}(9)$. Let $i: P \rightarrow \mathrm{SL}_{2}(9)$ be the inclusion. We have $i_{*}: H_{2}(P) \rightarrow H_{2}\left(\mathrm{SL}_{2}(9)\right)$ and the map called transfer $t: H_{2}\left(\mathrm{SL}_{2}(9)\right) \rightarrow$ $H_{2}(P)$. It is well known that the composition $i_{*} \circ t$ is multiplication by the index of $P$ in $\mathrm{SL}_{2}(9), 80$ in this case. Since 80 is relative prime with 3 , we have that $i_{*} \circ t$ is an isomorphism. Since $H_{2}(P) \simeq \mathbb{Z}_{3}$, this means that $i_{*}$ is an isomorphism. Thus $H_{2}\left(\mathrm{SL}_{2}(9)\right)$ is generated by the element $B \wedge A$ (see section 2.5). We need an element in $H_{2}(S)$ that is mapped to $B \wedge A$. Since $\langle B\rangle \in \mathcal{F}$, we have a generator $\widehat{B} \in S$, corresponding to $B$. Of course $\varphi(\widehat{B})=B$. A proof very similar to that of Lemma 1.1.5 shows that $\varphi^{-1}\left(C_{S L_{2}(9)}(B)\right)=C_{S}(\widehat{B})$. Choose an element $\widehat{A} \in S$ with $\varphi(\widehat{A})=A$. Then $\widehat{A}$ and $\widehat{B}$ commute in $S$, and the element $\widehat{B} \wedge \widehat{A}$ in $H_{2}(S)$ is mapped to $B \wedge A$. Thus $H_{2} S \rightarrow H_{2} \mathrm{SL}_{2}(9)$ is an epimorphism. Therefore $\varphi: S \rightarrow \mathrm{SL}_{2}(9)$ is an isomorphism.

\subsection{Covering groups}

We begin with an extension

$$
K \hookrightarrow \widehat{G} \stackrel{\pi}{\longrightarrow} G,
$$

of finite groups. Our first lemma is: 
Lemma 3.11. If the extension (3) is central, then for every subgroup $F$ of $G$, whose order is relatively prime with $|K|$, there is a unique homomorphism $\theta: F \rightarrow \widehat{G}$ such that $\pi \circ \theta$ equals the inclusion $F \rightarrow G$.

Proof. The central extension (3) induces the central extension

$$
K \longrightarrow \pi^{-1} F \longrightarrow F \text {. }
$$

Since $(|F|,|K|)=1$, the Schur-Zassenhaus' theorem [14], pg. 211, tells us that $\pi^{-1} F \simeq$ $K \times F$. There is a unique homomorphism $F \rightarrow \pi^{-1} F$ such that the diagram

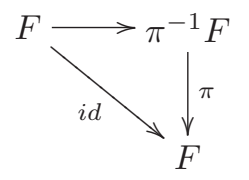

commutes. The homomorphism we are looking for is then

$$
F \longrightarrow \pi^{-1} F^{C} \widehat{G} \text {. }
$$

Suppose now that we have a generating active family $\mathcal{F}=\left\{F_{i}\right\}_{i \in I}$ of subgroups of $G$, such that $\left(\left|F_{i}\right|,|K|\right)=1$ for every $i \in I$. According to the above lemma, there are uniquely determined subgroups $\widehat{F}_{i}$ of $\widehat{G}$ such that $\left.\pi\right|_{\widehat{F}_{i}}: \widehat{F}_{i} \rightarrow F_{i}$ is an isomorphism. Since conjugation does not alter order we have that $\widehat{\mathcal{F}}:=\left\{\widehat{F}_{i}\right\}_{i \in I}$ is an active family, with inclusions as in the family $\mathcal{F}$. We call such a family $\widehat{\mathcal{F}}$ a lifting of the family $\mathcal{F}$ to $\widehat{G}$. It is clear that the active sum of the family $\mathcal{F}$ is isomorphic to the active sum of the family $\widehat{\mathcal{F}}$. Now we have:

Proposition 3.12. Assume that the extension (3) is stem, then, with the above notation, $\widehat{\mathcal{F}}$ is a generating active family of subgroups of $\widehat{G}$

Proof. Let $N$ be the subgroup of $\widehat{G}$ generated by $\widehat{\mathcal{F}}$. Since $\widehat{\mathcal{F}}$ is active and $K$ is central, the subgroup $N$ is a normal subgroup of $\widehat{G}$. It is not hard to see that $\widehat{G}=K N$. It follows that $\widehat{G} / N=K N / N \simeq K /(K \cap N)$. Since the extension is central we have that $K /(K \cap N)$ is abelian. Therefore $\widehat{G}^{\prime} \subseteq N$. Since the extension is stem, we have that $K \subseteq \widehat{G}^{\prime} \subseteq N$. Therefore $N=\widehat{G}$.

An immediate consequence of the previous two propositions is:

Proposition 3.13. If (3) is a stem extension and $\mathcal{F}$ is an active generating family of subgroups of $G$ such that, for every $F \in \mathcal{F}$ the order of $F$ is relatively prime with the order of $K$, then there is an epimorphism from the active sum of the family $\mathcal{F}$ to $\widehat{G}$.

We relate this results with the Schur multiplier: 
Theorem 3.14. Let $G$ be a finite group. Let $p$ be a prime dividing the order of $H_{2} G$. If $G$ is the active sum of an active generating family $\mathcal{F}$ of subgroups of $G$, then there exists an $F \in \mathcal{F}$ such that $p$ divides the order of $F$.

Proof. Assume $\mathcal{F}$ is an active generating family of subgroups of $G$. Let $S$ be the active sum of $\mathcal{F}$. Assume that $p$ does not divide the order of any element of $\mathcal{F}$. Let $\widehat{G}$ be a covering group of $G$, then we have a stem extension $H_{2} G>\mu \stackrel{{ }^{\mu}}{\longrightarrow} \stackrel{\pi}{\longrightarrow} G$. Since $p|| H_{2} G \mid$ and $H_{2} G$ is abelian, there is a subgroup $N$ of $H_{2} G$ such that $H_{2} G / N \simeq \mathbb{Z}_{p}$. Since the above extension is stem, the image of $N$ is normal in $\widehat{G}$. We obtain another stem extension $H_{2} G / N>\mu \stackrel{G}{\longrightarrow} / \mu(N) \stackrel{\pi}{\longrightarrow} G$, that is to say, a stem extension of the form:

$$
\mathbb{Z}_{p}>H \longrightarrow G \text {. }
$$

By what was done in the above propositions, taking into account the fact that $p$ does not divide any element of $\mathcal{F}$, there is a unique lifting $\widehat{\mathcal{F}}$ of $\mathcal{F}$ to $H$, and thus, an epimorphism $S \rightarrow H$. Since $|H|=|G| p$, we conclude that $S$ is not isomorphic to $G$.

Theorem 3.15. Let $G$ be a finite group and $\widehat{G}$ a covering group of $G$. If $\mathcal{F}$ is a regular and independent generating active family of subgroups of $G$ such that for every $F \in \mathcal{F}$ we have that $|F|$ and $\left|H_{2} G\right|$ are relatively prime, then the active sum of $\mathcal{F}$ is isomorphic to $\widehat{G}$.

Proof. According to Proposition 3.13, the active sum $S$ of the family $\mathcal{F}$ is projected onto $\widehat{G}$. Let $\varphi: S \rightarrow G$ be the usual projection and $K:=\operatorname{ker} \varphi$. Consider the following diagram

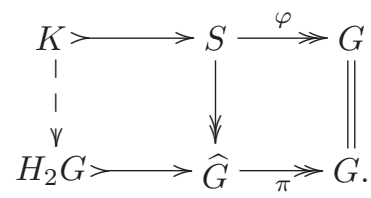

Since the right hand square commutes, we can induce the dotted arrow $\mathrm{K} \rightarrow \mathrm{H}_{2} \mathrm{G}$, making the diagram commute. Since $S$ projects onto $\widehat{G}$, we have that $|\widehat{G}| \leq|S|$. Consider the exact sequence

$$
\mathrm{H}_{2} \mathrm{~S} \stackrel{\varphi_{*}}{\longrightarrow} \mathrm{H}_{2} \mathrm{G} \longrightarrow \mathrm{K} \longrightarrow \mathrm{H}_{1} \mathrm{~S} \stackrel{\varphi_{*}}{\longrightarrow} \mathrm{H}_{1} \mathrm{G} \longrightarrow 0
$$

Since $\mathcal{F}$ is regular and independent, we have that $\varphi_{*}$ is an isomorphism at the $H_{1}$ level. This means that the next arrow to the left is zero. Therefore the arrow $H_{2} G \rightarrow K$ is an epimorphism. Thus $|K| \leq\left|H_{2} G\right|$. Now, it is just a question of cardinality:

$$
|S|=|K||G| \leq\left|H_{2} G\right||G|=|\widehat{G}| \leq|S| \text {. }
$$

Therefore $S$ is isomorphic to $\widehat{G}$.

Conjecture. Every covering group of a finite simple group is an active sum of cyclic subgroups. 


\section{References}

[1] M. Aschbacher, 3-transposition groups, Cambridge Tracts in Mathematics, vol. 124, Cambridge University Press, Cambridge, 1997, ISBN 0-521-57196-0.

[2] R. Baer, Free sums of groups and their generalizations. An analysis of the associative law, Amer. J. Math. 71 (1949), 706-742.

[3] J. A. Belana and F. Tomàs, 1996, without title (personal communication).

[4] F. R. Beyl and J. Tappe, Group extensions, representations, and the Schur multiplicator, Lecture Notes in Mathematics, vol. 958, Springer-Verlag, Berlin, 1982, ISBN 3-540-11954-X.

[5] P. Burmeister, Partial algebras - an introductory survey, Algebras and Orders (Montreal, PQ, 1991), NATO Adv. Sci. Inst. Ser. C Math. Phys. Sci., vol. 389, Kluwer Acad. Publ., Dordrecht, 1993, pp. 1-70.

[6] A. J. Díaz-Barriga, Reconstruction of profinite groups from the closed normal hulls of its Sylow subgroups and natural actions, J. Algebra 103 (1986), 600-605.

[7] A. J. Díaz-Barriga and L. Y. López, Active sums of pro-C groups, An. Inst. Mat. Univ. Nac. Autónoma México 19 (1979), 21-39. (English, with Spanish summary)

[8] A. J. Díaz-Barriga and L. Román, Note on a result of Puig-Ribenboim, C. R. Math. Rep. Acad. Sci. Canada 7 (1985), 361-362.

[9] B. Eckmann, P. J. Hilton, and U. Stammbach, On the homology theory of central group extensions. I. The commutator map and stem extensions, Comment. Math. Helv. 47 (1972), 102122.

[10] Roger Fenn and Colin Rourke, Racks and links in codimension two, J. Knot Theory Ramifications 1 (1992), 343-406.

[11] T. A. Fournelle and K. W. Weston, Generalizations of Steinberg groups, Series in Algebra, vol. 4, World Scientific Publishing Co. Inc., River Edge, NJ, 1996, ISBN 981-02-2028-6.

[12] T. Ganea, Homologie et extensions centrales de groupes, C. R. Acad. Sci. Paris Sér. A-B 266 (1968), A556-A558. (French)

[13] S. Gervais, Presentation and central extensions of mapping class groups, Trans. Amer. Math. Soc. 348 (1996), 3097-3132.

[14] D. Gorenstein, Finite groups, Harper \& Row Publishers, New York, 1968.

[15] _ Finite simple groups, University Series in Mathematics, Plenum Publishing Corp., New York, 1982, ISBN 0-306-40779-5.

[16] J. Harer, The second homology group of the mapping class group of an orientable surface, Invent. Math. 72 (1983), 221-239.

[17] A. Hatcher and W. Thurston, A presentation for the mapping class group of a closed orientable surface, Topology 19 (1980), 221-237.

[18] H. Herrlich and G. E. Strecker, Category theory, Sigma Series in Pure Mathematics, vol. 1, Heldermann Verlag, Berlin, 1979, ISBN 3-88538-001-3.

[19] A. Herschfeld, The equation $2^{x}-3^{y}=d$, Bull. Amer. Math. Soc. 42 (1936), 231-234.

[20] I. N. Herstein, Topics in algebra, Blaisdell Publishing Co. Ginn and Co. New York-TorontoLondon, 1964.

[21] P. J. Hilton and U. Stammbach, A course in homological algebra, Springer-Verlag, New York, 1971.

[22] J. E. Humphreys, Reflection groups and Coxeter groups, Cambridge Studies in Advanced Mathematics, vol. 29, Cambridge University Press, Cambridge, 1990, ISBN 0-521-37510-X. 
[23] G. Karpilovsky, The Schur multiplier, London Mathematical Society Monographs. New Series, vol. 2, The Clarendon Press Oxford University Press, New York, 1987, ISBN 0-19-853554-6.

[24] M. A. Kervaire, On higher dimensional knots, Differential and Combinatorial Topology (A Symposium in Honor of Marston Morse), Princeton Univ. Press, Princeton, N.J., 1965, pp. 105119

[25] R. C. Kirby, A calculus for framed links in $S^{3}$, Invent. Math. 45 (1978), 35-56.

[26] P. Kutzko, On groups of finite weight, Proc. Amer. Math. Soc. 55 (1976), 279-280.

[27] J. C. Lennox and J. Wiegold, Generators and killers for direct and free products, Arch. Math. (Basel) 34 (1980), 296-300.

[28] W. B. R. Lickorish, A representation of orientable combinatorial 3-manifolds, Ann. of Math. (2) 76 (1962), 531-540.

[29] N. Lu, A simple proof of the fundamental theorem of Kirby calculus on links, Trans. Amer. Math. Soc. 331 (1992), 143-156.

[30] R. C. Lyndon and P. E. Schupp, Combinatorial group theory, Springer-Verlag, Berlin, 1977, ISBN 3-540-07642-5.

[31] S. MacLane, Categories for the working mathematician, Springer-Verlag, New York, 1971.

[32] F. Marmolejo, Comparación entre definiciones de suma activa, 1987, bachelor degree thesis.

[33] F. Marmolejo and L. Román, On active sums, XXIIIrd National Congress of the Mexican Mathematical Society (Spanish) (Guanajuato, 1990), Aportaciones Mat. Comun., vol. 9, Soc. Mat. Mexicana, México, 1991, pp. 181-197.

[34] P. Paredes and F. Tomás, The intersections of normal subgroups generated by unions of Sylow subgroups, An. Inst. Mat. Univ. Nac. Autónoma México 17 (1977), 87-93.

[35] P. Ribenboim, Active sums of groups, J. Reine Angew. Math. 325 (1981), 153-182.

[36] _ Active sums of profinite groups, Bol. Soc. Brasil. Mat. 14 (1983), 125-132.

[37] D. J. S. Robinson, A course in the theory of groups, Graduate Texts in Mathematics, vol. 80, Springer-Verlag, New York, 1982, ISBN 0-387-90600-2.

[38] J. J. Rotman, An introduction to homological algebra, Pure and Applied Mathematics, vol. 85, Academic Press Inc. [Harcourt Brace Jovanovich Publishers], New York, 1979, ISBN 0-12599250-5.

[39] J. Simon, Wirtinger approximations and the knot groups of $F^{n}$ in $S^{n+2}$, Pacific J. Math. 90 (1980), 177-190.

[40] J. Stallings, Group theory and three-dimensional manifolds, Yale University Press, New Haven, Conn., 1971.

[41] M. Suzuki, On a class of doubly transitive groups, Ann. of Math. (2) 75 (1962), 105-145.

[42] F. Tomás, Sobre los normalizados de los grupos de descomposición, An. Inst. Mat. Univ. Nac. Autónoma México 13 (1973), 187-208.

[43] __ Un análogo de suma directa para sistemas de subgrupos normales, An. Inst. Mat. Univ. Nac. Autónoma México 13 (1973), 161-186.

[44] _ Reconstrucción de los grupos finitos a partir de las cerraduras normales de sus subgrupos de Sylow y sus acciones mutuas, An. Inst. Mat. Univ. Nac. Autónoma México 18 (1978), 29-49 (1979).

[45] B. Wajnryb, A simple presentation for the mapping class group of an orientable surface, Israel J. Math. 45 (1983), 157-174. 